\title{
An Efficient Method for Mitigating Longevity Value-at-Risk
}

\author{
Yanxin Liu* and Johnny Siu-Hang $\mathrm{Li}^{\dagger}$
}

\begin{abstract}
Many of the existing index-based longevity hedging strategies focus on the reduction in variance. However, solvency capital requirements are typically based on the $\tau$-year-ahead Value-at-Risk, with $\tau=1$ under Solvency II. Optimizing a longevity hedge using variance minimization is particularly inadequate when the cost of hedging is non-zero and mortality improvements are driven by a skewed and/or heavy-tailed distribution. In this paper, we contribute a method to formulate a value hedge that aims to minimize the Value-at-Risk of the hedged position over a horizon of $\tau$ years. The proposed method works with all stochastic mortality models that can be formulated in a state-space form, even when a non-normal distributional assumption is made. We further develop a technique to expedite the evaluation of a value longevity hedge. By utilizing the generic assumption that the innovations in the stochastic processes for the period and cohort effects are not serially correlated, the proposed technique spares us from the need for nested simulations that are generally required when evaluating a value hedge.
\end{abstract}

Keywords: Index-based longevity hedges; Solvency II; State-space models.

\section{Introduction}

To annuity providers, the biggest motivation to hedge longevity risk exposures is to reduce the amount of solvency capital they are required to hold. Solvency capital requirements vary across regulatory regimes, but the principles behind are similar. For Solvency II, whenever an internal model is used, the solvency capital requirement should be drawn from the Value-at-Risk of the hedger's liabilities over a horizon of one year at the $99.5 \%$ confidence level (see, e.g., Barrieu et al., 2012). When contemplating an index-based longevity hedging solution, it is natural for an annuity provider to ask: "how should the hedge be formulated so that my solvency capital requirement (Value-at-Risk at a certain confidence level) is minimized?" This paper is written to address this fundamental question, which we believe is still an open one to date.

\footnotetext{
*Yanxin Liu is an Assistant Professor in the Department of Finance at the University of Nebraska-Lincoln. Email: yanxin.liu@unl.edu.

${ }^{\dagger}$ Johnny Siu-Hang Li is Professor of Actuarial Studies at The University of Melbourne and Professor of Actuarial Science at the University of Waterloo. Email: johnny.li@unimelb.edu.au, shli@uwaterloo.ca.
} 
Longevity risk under solvency regimes has been studied by Börger (2010), Börger et al. (2014), Olivieri and Pitacco (2009) and Plat (2009a, 2011). These researchers have contributed stochastic mortality models which they deem to be well suited for the purpose of solvency capital calculations. For instance, Plat (2011) developed a three-factor mortality trend model, which he argued to be more appropriate for determining the one-year Value-at-Risk compared to models that assume a fixed long-term trend. Their contributions offer practitioners additional modeling choices for their solvency capital calculations, but they are not very much connected to the hedging front.

Some researchers have attempted to link Value-at-Risk to the context of longevity risk. For instance, Boyer et al. (2014), Dowd et al., (2006), Fujisawa and Li (2010, 2012) and Zhou et al., (2014) used Value-atRisk as a metric to quantify the longevity risk affecting insurers, pension plan providers and governments. Attempts have also been made to utilize Value-at-Risk as a metric to quantify the effectiveness of indexbased longevity hedges (e.g., Blake et al., 2014; Coughlan et al., 2011; Li and Hardy, 2011; Li and Luo, 2012; Plat, 2009a). We stress that in these previous attempts, Value-at-Risk is used solely at the measurement level, but is not involved in formulating any index-based longevity hedge.

On the other hand, existing hedging strategies are inadequate when the hedger's objective is to minimize the $\tau$-year (with $\tau=1$ under Solvency II) Value-at-Risk of the hedged position. Existing hedging strategies that are based on sensitivity measures such as key q-duration and longevity Greeks (e.g., Cairns, 2011, 2013; Li and Hardy, 2011; Li and Luo, 2012; Lin and Tsai, 2013; Zhou and Li, 2017a,b) allow a hedger to create a 'replicating portfolio' using index-based hedging instruments. Such a 'replicating portfolio' has a value that varies with the underlying mortality experience similarly to the liability being hedged, but generally it does not minimize the $\tau$-year ahead Value-at-Risk of the hedged position. Those that are derived using variance minimization (e.g., Liu and Li, 2017a,b; Zhang et al., 2017) are even more inadequate. As we demonstrate in this paper, the solutions to variance minimization and Value-at-Risk minimization are equivalent only under some special circumstances. Generally speaking, if the hedger's objective is to minimize Value-at-Risk, then variance minimization would lead to a sub-optimal hedging strategy and may result in a Value-at-Risk that is even higher than that of the unhedged position. It is also worth noting that the mentioned hedging strategies do not take the cost of the hedging into account. This treatment may be acceptable when minimizing variance (which measures only on the dispersion around the expected portfolio value), but is not when minimizing Value-at-Risk (which is sensitive to the expected portfolio value that depends on the costs of the hedging instruments).

When considering Value-at-Risk, it is important to make a prudent provision for extreme outcomes, as otherwise the riskiness of a portfolio may be understated. Nonetheless, more often than not, a normality assumption is made for the distributions of the innovations of the processes for the period and/or cohort effects in stochastic mortality models. Notable exceptions are the work of Ahmadi and Gaillardetz (2014), who considered four innovation distributions with skewness and/or excess kurtosis for the CairnsBlake-Dowd model, and the work of Wang et al. (2011), who considered five skewed and/or heavy-tailed innovation distributions for the Lee-Carter model. While these two studies demonstrated the benefit of using an appropriate non-normal distributional assumption in terms of goodness-of-fit to historical data, the impact of using a non-normal distributional assumption on longevity hedges (especially those that are developed to minimize Value-at-Risk) is yet to be investigated.

Complicating the research question further is the potential computational burden entailed. To simulate the $\tau$-year Value-at-Risk for a range of possible outcomes, nested simulations are generally required because for each simulated outcome another round of simulations is needed to calculate the time- $\tau$ portfolio value. 
One possible approach to avoid this problem is to use forward mortality models (e.g., Bauer et al., 2010), but this approach is not without criticisms. As Plat (2011) pointed out, forward mortality models are quite complex and not very transparent, and more importantly, they do not seem to fit the 'real world' setting in the calculation of the $\tau$-year Value-at-Risk. Another approach is to apply some sort of approximations, as suggested by Cairns (2011) and Plat (2011). Recently, Richards et al. (2014) proposed a Value-at-Risk calculation framework that requires neither nested simulations nor approximations, but the application of their method to longevity risk hedging is untested.

In this paper, we attempt to fill the aforementioned gaps in the literature by contributing a hedging strategy that aims to minimize the $\tau$-year Value-at-Risk of the hedged position at a given confidence level. Drawing on the recent work of Liu and Li (2017b), who developed a variance-minimizing hedging strategy for use with Model M7, we build our hedging strategy by utilizing the linkage between the hedged position to the innovations in the processes for the period and/or cohort effects in the assumed stochastic mortality model. In addition to the objective, a notable difference from the previous work of Liu and $\mathrm{Li}$ (2017b) is that we build our hedging strategy on the general state-space representation of stochastic mortality models (Fung et al., 2017). This foundation enables the resulting hedging strategy to be applicable readily to a wide range of stochastic mortality models, including but not limited to the Lee-Carter model (Lee and Carter, 1992), the Renshaw-Haberman model (Renshaw and Haberman, 2003), the Cairns-Blake-Dowd model and its variants (Cairns et al, 2006, 2009), and the Plat model (Plat, 2009b). We believe that this flexibility is important, as Solvency II allows insurers to use any internal model that passes the Internal Model Approval Process (IMAP).

We present three levels of theoretical results. In the first level, we make the usual assumption that the innovations in the period and cohort effect processes are normally distributed. The solution in the first level is transparent, allowing us to visualize the connection between Value-at-Risk and variance minimizations easily. Then, in the second level, we assume less restrictively that the innovations follow a member distribution in the family of (multivariate) stable elliptical distributions, a family of distributions that encompasses a wide range of symmetric distributions including Student's $t$ (which is heavier tailed than normal). Finally, in the third level, we assume that the innovations follow Azzalini and Capitanio's (2003) (multivariate) skew- $t$ distribution, a distribution that incorporates both skewness and excess kurtosis. For all three levels, we provide a analytical expression of the first-order condition for the optimization problem.

We incorporate the cost of hedging into the derivation of the optimal hedging strategy. Following Loeys et al. (2007), we consider a pricing formula that is linked to an assumed annualized Sharpe ratio, which measures the compensation demanded by the counterparty who takes longevity risk exposures from the hedger. The resulting optimal hedging strategy depends on the annualized Sharpe ratio. More interestingly, we show that the solution to the Value-at-Risk minimization ceases to exist if the annualized Sharpe ratio becomes too large (i.e., the cost of hedging becomes too high). Our results strengthen the work of Ngai and Sherris (2011), who examined empirically the risk reduction produced by q-forwards for different assumed annualized Sharpe ratios, and also the work of Levantesi and Menzietti (2017), who identified empirically the maximum market price of longevity risk that a hedger can withstand.

When evaluating the proposed longevity hedge (i.e., calculating the $\tau$-year Value-at-Risk of the hedged position), we are subject to the potential computational burden mentioned earlier. To mitigate the burden, we develop a more efficient simulation procedure by considering that (1) the innovations of the period and cohort effect processes are the only random components that are relevant to the trend risk surrounding the hedged position, and that (2) the innovations of the period and cohort effect processes are always assumed 
to be serially uncorrelated. In effect, the proposed simulation procedure reduces the required number of simulated sample paths from $M_{1} \times M_{2}$ (where $M_{1}$ and $M_{2}$ are both large numbers) to $M_{1}$ only. As with the Value-at-Risk framework of Richards et al. (2014), our proposed simulation procedure does not require any approximation.

The remainder of this paper is organized as follows. Section 2 presents the general state-space form of stochastic mortality models. Section 3 outlines our set-up. Section 4 details the derivation of our proposed hedging strategy in a general state-space setting, and illustrate the proposed hedging strategy with three example models that are commonly used in practice. Section 5 describes our proposed innovation-based simulation method, and explains how it can eliminate the need for nested simulations. Section 6 is devoted to the real-data illustrations. Finally, Section 7 concludes the paper.

\section{Stochastic Mortality-Models in a State-Space Form}

\subsection{The General State-Space Form}

Most discrete-time stochastic mortality models can be expressed in a state-space form. The general statespace representation of stochastic mortality models is composed of an observation equation and a transition equation. The former links the (transformed) age-specific death rates/probabilities to the underlying period and/or cohort effects, whereas the latter captures the random evolution of the underlying period and/or cohort effects.

The observation equation can be written as follows:

$$
\vec{y}_{t}=\vec{d}+\mathbf{B} \vec{\alpha}_{t}+\vec{\epsilon}_{t}
$$

where $\vec{y}_{t}$ is the vector of observations (the vector of transformed age-specific death rates/probabilities), $\vec{d}$ is a constant vector (which typically contains the age-effect parameters), $\vec{\alpha}_{t}$ is the vector of hidden states (the underlying period and/or cohort effects), $\mathbf{B}$ is the design matrix (which governs how the observations are related to the hidden states) and $\vec{\epsilon}_{t}$ is the vector of sampling errors at time $t$.

The transition equation characterizes the random evolution of the vector of hidden states through a first-order Markov process:

$$
\vec{\alpha}_{t}=\vec{c}+\mathbf{A} \vec{\alpha}_{t-1}+\vec{\eta}_{t}^{*}
$$

where $\vec{c}$ is a constant vector (the vector of drifts), $\mathbf{A}$ is the transition matrix that captures the serial dependence of the hidden states, and $\vec{\eta}_{t}^{*}$ is the vector of random innovations. It is assumed that $\left\{\vec{\eta}_{t}^{*}\right\}$ is a sequence of identically distributed and serially uncorrelated random vectors, each of which has a zero mean vector and a variance-covariance matrix of $\mathbf{Q}$.

For models with cohort effects, $\vec{\eta}_{t}^{*}$ may be a degenerate vector (i.e., some of the elements in $\vec{\eta}_{t}^{*}$ are always zero). To facilitate the derivation of our hedging strategy (which is based on the sensitivity to the innovations), we use $\vec{\eta}_{t}$ to represent the non-degenerate portion of $\vec{\eta}_{t}^{*}$. We can relate $\vec{\eta}_{t}$ and $\vec{\eta}_{t}^{*}$ using the following equation:

$$
\vec{\eta}_{t}^{*}=\mathbf{C} \vec{\eta}_{t}
$$


where $\mathbf{C}$ is an appropriately defined matrix of which the number of rows equals the length of $\vec{\eta}_{t}^{*}$ and the number of columns equals the length of $\vec{\eta}_{t}$. Accordingly, the transition equation can be rewritten as

$$
\vec{\alpha}_{t}=\vec{c}+\mathbf{A} \vec{\alpha}_{t-1}+\mathbf{C} \vec{\eta}_{t}
$$

In this stage, we do not need to make any distributional assumptions on $\vec{\epsilon}_{t}$ and $\vec{\eta}_{t}$. It is important to note that our proposed hedging strategy does not require $\vec{\eta}_{t}$ to follow a (multivariate) normal distribution, thereby allowing the hedger to better optimize his/her longevity hedge when the distribution of $\vec{\eta}_{t}$ exhibits skewness and/or excess kurtosis.

\subsection{Example Models}

Our proposed hedging strategy can be applied to any stochastic mortality model that can be written in a state-space form. To illustrate our proposed hedging strategy, we consider three example models: the Cairns-Blake-Dowd model (Cairns et al., 2006), the Lee-Carter model (Lee and Carter, 1992) and the Renshaw- Haberman model (Renshaw and Haberman, 2003). In the rest of this section, we demonstrate how each of the three example models can be expressed in a state-space form.

\subsubsection{The Cairns-Blake-Dowd Model Expressed in a State-Space Form}

Let $q_{x, t}$ be the observed probability that an individual dies between time $t-1$ and $t$ (i.e., during year $t$ ), given that he/she has survived to age $x$ at time $t-1$. The Cairns-Blake-Dowd model assumes that

$$
\operatorname{logit}\left(q_{x, t}\right)=\ln \left(\frac{q_{x, t}}{1-q_{x, t}}\right)=k_{t}^{(1)}+k_{t}^{(2)}(x-\bar{x})+\epsilon_{x, t}
$$

where $\bar{x}$ is the mid-point of the age range $\left[x_{a}, x_{b}\right]$ to which the model is calibrated, $k_{t}^{(1)}$ and $k_{t}^{(2)}$ are the time- $t$ values of the period (time-related) effects, $\epsilon_{x, t}$ is the sampling error for age $x$ and year $t$. Typically, the evolution of the period effects is captured by a bivariate random walk with constant drifts,

$$
\left\{\begin{array}{c}
k_{t}^{(1)}=c^{(1)}+k_{t-1}^{(1)}+\eta_{t}^{(1)} \\
k_{t}^{(2)}=c^{(2)}+k_{t-1}^{(2)}+\eta_{t}^{(2)}
\end{array},\right.
$$

where $c^{(1)}$ and $c^{(2)}$ are the constant drifts, and $\eta_{t}^{(1)}$ and $\eta_{t}^{(2)}$ are the time- $t$ innovations. For any $t, \eta_{t}^{(1)}$ and $\eta_{t}^{(2)}$ follow a bivariate distribution with a zero mean vector and a constant variance-covariance matrix. It is assumed that for $i=1,2, \eta_{s}^{(i)}$ and $\eta_{u}^{(i)}$ are uncorrelated when $s \neq u$.

To express the Cairns-Blake-Dowd model in a state-space form, we first obtain the observation equation (equation (2.1)) by considering (2.4) for all $x=x_{a}, \ldots, x_{b}$ jointly and setting

$$
\vec{y}_{t}=\left(\begin{array}{c}
\operatorname{logit}\left(q_{x_{a}, t}\right) \\
\operatorname{logit}\left(q_{x_{a}+1, t}\right) \\
\vdots \\
\operatorname{logit}\left(q_{x_{b}, t}\right)
\end{array}\right), \quad \vec{d}=\left(\begin{array}{c}
0 \\
0 \\
\vdots \\
0
\end{array}\right), \quad \mathbf{B}=\left(\begin{array}{cc}
1 & \left(x_{a}-\bar{x}\right) \\
1 & \left(x_{a}+1-\bar{x}\right) \\
\vdots & \vdots \\
1 & \left(x_{b}-\bar{x}\right)
\end{array}\right), \quad \vec{\alpha}_{t}=\left(\begin{array}{c}
k_{t}^{(1)} \\
k_{t}^{(2)}
\end{array}\right) \quad \text { and } \quad \vec{\epsilon}_{t}=\left(\begin{array}{c}
\epsilon_{x_{a}, t} \\
\epsilon_{x_{a}+1, t} \\
\vdots \\
\epsilon_{x_{b}, t}
\end{array}\right) .
$$


We then obtain the transition equation (equation (2.2)) by setting

$$
\vec{\alpha}_{t}=\left(\begin{array}{c}
k_{t}^{(1)} \\
k_{t}^{(2)}
\end{array}\right), \quad \vec{c}=\left(\begin{array}{c}
c^{(1)} \\
c^{(2)}
\end{array}\right), \quad \mathbf{A}=\left(\begin{array}{ll}
1 & 0 \\
0 & 1
\end{array}\right), \quad \vec{\eta}_{t}^{*}=\left(\begin{array}{l}
\eta_{t}^{(1)} \\
\eta_{t}^{(2)}
\end{array}\right) \quad \text { and } \quad \mathbf{Q}=\left(\begin{array}{ll}
Q_{11} & Q_{12} \\
Q_{21} & Q_{22}
\end{array}\right),
$$

where $Q_{1,1}$ represents the variance of $\eta_{t}^{(1)}, Q_{2,2}$ represents the variance of $\eta_{t}^{(2)}$, and $Q_{1,2}=Q_{2,1}$ represents the covariance between $\eta_{t}^{(1)}$ and $\eta_{t}^{(2)}$.

In this model, $\vec{\eta}_{t}^{*}$ is a non-degenerate vector. Hence, we have $\vec{\eta}_{t}=\vec{\eta}_{t}^{*}$ and $\mathbf{C}$ in the modified transition equation (equation (2.3)) being a $2 \times 2$ identity matrix.

\subsubsection{The Lee-Carter Model in a State-Space Form}

Let $m_{x, t}$ be the observed central rate of death for age $x$ and year $t$. The Lee-Carter model models assumes that

$$
\ln \left(m_{x, t}\right)=a_{x}+b_{x} k_{t}+\epsilon_{x, t}
$$

where $a_{x}$ and $b_{x}$ are the age-specific parameters, $k_{t}$ is the time- $t$ value of the period effect, and $\epsilon_{x, t}$ is the sampling error for age $x$ and year $t$. Typically, the evolution of the period effect is captured by a (univariate) random walk with a constant drift:

$$
k_{t}=c+k_{t-1}+\eta_{t}
$$

where $c$ is the drift and $\eta_{t}$ is the innovation at time $t$. It is assumed that $\left\{\eta_{t}\right\}$ is a sequence of independent and identically distributed random variables with a zero mean and constant variance.

The Lee-Carter model can be expressed in a state-space form straightforwardly. For the observation equation, we set

$$
\vec{y}_{t}=\left(\begin{array}{c}
\ln \left(m_{x_{a}, t}\right) \\
\ln \left(m_{x_{a}+1, t}\right) \\
\vdots \\
\ln \left(m_{x_{b}, t}\right)
\end{array}\right), \quad \vec{d}=\left(\begin{array}{c}
a_{x_{a}} \\
a_{x_{a}+1} \\
\vdots \\
a_{x_{b}}
\end{array}\right), \quad \mathbf{B}=\left(\begin{array}{c}
b_{x_{a}} \\
b_{x_{a}+1} \\
\vdots \\
b_{x_{b}}
\end{array}\right), \quad \vec{\alpha}_{t}=k_{t} \quad \text { and } \quad \vec{\epsilon}_{t}=\left(\begin{array}{c}
\epsilon_{x_{a}, t} \\
\epsilon_{x_{a}+1, t} \\
\vdots \\
\epsilon_{x_{b}, t}
\end{array}\right)
$$

where $x_{a}$ and $x_{b}$ are the first and last ages in the age range to which the model is calibrated, respectively. For the transition equation, we set

$$
\vec{\alpha}_{t}=k_{t}, \quad \vec{c}=c, \quad \mathbf{A}=1, \quad \vec{\eta}_{t}^{*}=\eta_{t} \quad \text { and } \quad \mathbf{Q}=Q
$$

where $Q$ represents the variance of $\eta_{t}$.

In this model, $\vec{\eta}_{t}^{*}$ is a scalar. Hence, for the modified transition equation, we have

$$
\mathbf{C}=1 \quad \text { and } \quad \vec{\eta}_{t}^{*}=\vec{\eta}_{t}=\eta_{t}
$$




\subsubsection{The Renshaw-Haberman Model in a State-Space Form}

The Renshaw-Haberman model assumes that the natural logarithm of the central death rate at age $x$ and year $t$ is structured as follows:

$$
\ln \left(m_{x, t}\right)=a_{x}+b_{x}^{(k)} k_{t}+b_{x}^{(g)} g_{t-x}+\epsilon_{x, t}
$$

where $a_{x}, b_{x}^{(k)}$ and $b_{x}^{(g)}$ are age-specific parameters, $k_{t}$ is the time- $t$ value of the period effect, $g_{t-x}$ is the cohort effect for year-of-birth $t-x$, and $\epsilon_{x, t}$ is the sampling error for age $x$ and year $t$.

The evolution of the period effect is typically assumed to follow a (univariate) random walk with drift,

$$
k_{t}=c^{(k)}+k_{t-1}+\eta_{t}^{(k)}
$$

where $c^{(g)}$ is the drift for the period effect and $\eta_{t}^{(k)}$ is the time- $t$ innovation for the period effect. It is often not easy to identify a suitable process for the cohort effect. For the purpose of illustration, we assume that the cohort effect follows a first-order autoregressive process,

$$
g_{t-x}=c^{(g)}+\phi_{1} g_{t-x-1}+\eta_{t-x}^{(g)}
$$

where $c^{(g)}$ is a constant and $\eta_{t-x}^{(g)}$ is the innovation for year-of-birth $t-x$. It is assumed that both $\left\{\eta_{t}^{(k)}\right\}$ and $\left\{\eta_{t-x}^{(g)}\right\}$ are sequences of independent and identically distributed random variables with a zero mean and constant variances.

Compared to the previous two models, the state-space form of the Renshaw-Haberman model is more sophisticated. To obtain the observation equation, we set

$$
\begin{aligned}
\vec{y}_{t}=\left(\begin{array}{c}
\ln \left(m_{x_{a}, t}\right) \\
\ln \left(m_{x_{a}+1, t}\right) \\
\vdots \\
\ln \left(m_{x_{b}-1, t}\right) \\
\ln \left(m_{x_{b}, t}\right)
\end{array}\right), \quad \vec{d}=\left(\begin{array}{c}
a_{x_{a}} \\
a_{x_{a}+1} \\
\vdots \\
a_{x_{b}-1} \\
a_{x_{b}}
\end{array}\right), \quad \mathbf{B}=\left(\begin{array}{cccccc}
b_{x_{a}}^{(k)} & b_{x_{a}}^{(g)} & 0 & \cdots & 0 & 0 \\
b_{x_{a}+1}^{(k)} & 0 & b_{x_{a}+1}^{(g)} & \cdots & 0 & 0 \\
\vdots & \vdots & \vdots & \ddots & \vdots & \vdots \\
b_{x_{b}-1}^{(k)} & 0 & 0 & \cdots & b_{x_{b}-1}^{(g)} & 0 \\
b_{x_{b}}^{(k)} & 0 & 0 & \cdots & 0 & b_{x_{b}}^{(g)}
\end{array}\right), \\
\vec{\alpha}_{t}=\left(\begin{array}{c}
\epsilon_{t} \\
g_{t-x_{a}, t} \\
g_{t-x_{a}-1} \\
\vdots \\
\epsilon_{x_{a}+1, t} \\
\vdots \\
\epsilon_{x_{b}-1, t} \\
\epsilon_{x_{b}, t}
\end{array}\right) \text { and } \vec{\epsilon}_{t}=\left(\begin{array}{ll} 
& \\
\end{array}\right)
\end{aligned}
$$

where $x_{a}$ and $x_{b}$ are the first and last ages in the age range to which the model is calibrated, respectively. 
To obtain the transition equation, we set

$$
\vec{\alpha}_{t}=\left(\begin{array}{c}
k_{t} \\
g_{t-x_{a}} \\
g_{t-x_{a}-1} \\
\vdots \\
g_{t-x_{b}+1} \\
g_{t-x_{b}}
\end{array}\right), \quad \vec{c}=\left(\begin{array}{c}
c^{(k)} \\
c^{(g)} \\
0 \\
\vdots \\
0 \\
0
\end{array}\right), \quad \mathbf{A}=\left(\begin{array}{cccccc}
1 & 0 & 0 & \cdots & 0 & 0 \\
0 & \phi_{1} & 0 & \cdots & 0 & 0 \\
0 & 1 & 0 & \cdots & 0 & 0 \\
0 & 0 & 1 & \cdots & 0 & 0 \\
\vdots & \vdots & \vdots & \ddots & \vdots & \vdots \\
0 & 0 & 0 & \cdots & 1 & 0
\end{array}\right), \quad \vec{\eta}_{t}^{*}=\left(\begin{array}{c}
\eta_{t}^{(k)} \\
\eta_{t-x_{a}}^{(g)} \\
0 \\
\vdots \\
0 \\
0
\end{array}\right)
$$

and

$$
\mathbf{Q}=\left(\begin{array}{ll}
Q_{11} & Q_{12} \\
Q_{21} & Q_{22}
\end{array}\right)
$$

where $Q_{1,1}$ represents the variance of $\eta_{t}^{(k)}, Q_{2,2}$ represents the variance of $\eta_{t-x_{a}}^{(g)}$, and $Q_{1,2}=Q_{2,1}$ represents the covariance between $\eta_{t}^{(k)}$ and $\eta_{t-x_{a}}^{(g)}{ }^{1}$ In the above, $\vec{\alpha}_{t}, \vec{c}$ and $\vec{\eta}_{t}^{*}$ are vectors with $\left(x_{b}-x_{a}+2\right)$ elements and $\mathbf{A}$ is a $\left(x_{b}-x_{a}+2\right)$-by- $\left(x_{b}-x_{a}+2\right)$ matrix.

In this model, $\vec{\eta}_{t}^{*}$ is a degenerate vector with all but the first two elements being always zero. To obtain the modified transition equation (equation (2.3)), we set

$$
\vec{\eta}_{t}=\left(\begin{array}{c}
\eta_{t}^{(k)} \\
\eta_{t-x_{a}}^{(g)}
\end{array}\right) \text { and } \mathbf{C}=\left(\begin{array}{cc}
1 & 0 \\
0 & 1 \\
0 & 0 \\
\vdots & \vdots \\
0 & 0 \\
0 & 0
\end{array}\right)
$$

\section{The Set-up}

In this section, we describe the liability being hedged and the hedging instruments, and explain how they are linked to a mortality model that is expressed in a state-space form.

Let us begin with some additional notation. We use $\mathbf{B}_{x}$. to denote the age- $x$-related row in matrix $\mathbf{B}$, and $y_{x, t}, d_{x}$ and $\epsilon_{x, t}$ to represent the age- $x$-related elements in vectors $\vec{y}_{t}, \vec{d}_{x}$ and $\vec{\epsilon}_{t}$, respectively; that is,

$$
y_{x, t}=d_{x}+\mathbf{B}_{x,}, \vec{\alpha}_{t}+\epsilon_{x, t}
$$

As with typical longevity hedging strategies, our proposed hedging strategy focuses only on trend risk (which arises from the random evolution of the hidden states). Sampling risk (which arises from the random observational errors) is excluded. Accordingly, when setting up the hedging strategy, we exclude $\epsilon_{x, t}$ for

\footnotetext{
${ }^{1}$ In the literature (including the original work of Renshaw and Haberman (2003)), $\eta_{t}^{(k)}$ and $\eta_{t-x_{a}}^{(g)}$ are typically assumed to be independent. Our state-space formulation permits the user to specify the dependence between $\eta_{t}^{(k)}$ and $\eta_{t-x_{a}}^{(g)}$, if he/she finds such dependence significant.
} 
any $x$ and $t$, and define

$$
\tilde{y}_{x, t}=d_{x}+\mathbf{B}_{x, \cdot} \cdot \vec{\alpha}_{t}
$$

which is subject to trend risk only. Similarly, we define $\tilde{m}_{x, t}$ and $\tilde{q}_{x, t}$ as the values of $m_{x, t}$ and $q_{x, t}$ when the observational error $\epsilon_{x, t}$ is set to zero. For example, when the Cairns-Blake-Dowd model is assumed, we have

$$
\operatorname{logit}\left(\tilde{q}_{x, t}\right)=\ln \left(\frac{\tilde{q}_{x, t}}{1-\tilde{q}_{x, t}}\right)=k_{t}^{(1)}+k_{t}^{(2)}(x-\bar{x}) .
$$

We can interpret $\tilde{m}_{x, t}$ and $\tilde{q}_{x, t}$ to mean the underlying (unobserved) death rate/probability for age $x$ and year $t$.

\subsection{The Liability Being Hedged}

Suppose that the hedge is established at time $t=t_{0}$ (i.e., the end of year $t_{0}$ ). The liability being hedged is a $T_{L}$-year deferred whole life annuity-due that is sold to individuals who are aged $x_{0}$ at time $t=t_{0}$. No payment is made during the deferment period (the first $T_{L}$ years). Starting in year $T_{L}+1$, a payment of $\$ 1$ is made at the beginning of each year to every surviving annuitant. The payment structure of the liability is illustrated in Figure 1.

Let $L$ be the sum of the per contract cash flows, measured in time- $t_{0}$ dollars. Ignoring sampling risk, we have

$$
L=\sum_{u=1}^{\omega-x_{0}-T_{L}}\left(e^{-r\left(T_{L}+u-1\right)}\left(\prod_{i=1}^{T_{L}+u-1} \tilde{p}_{x_{0}+i-1, t_{0}+i}\right)\right),
$$

where $r$ is the interest rate at which cash flows are discounted, and $\tilde{p}_{x, t}:=1-\tilde{q}_{x, t}$ represents the underlying probability that an individual survives to age $x$ at time $t$, given that he/she is alive at time $t-1$. The relationship between $\tilde{p}_{x, t}$ and $\tilde{y}_{x, t}$ depends on the assumed model. For models capturing $\operatorname{logit}\left(q_{x, t}\right)$ (e.g., the Cairns-Blake-Dowd model and its variants), we have

$$
\tilde{p}_{x, t}=\frac{1}{1+\exp \left(\tilde{y}_{x, t}\right)} .
$$

For models capturing $\ln \left(m_{x, t}\right)$ (e.g., the Lee-Carter model and the Renshaw-Haberman model), we have

$$
\tilde{p}_{x, t}=\exp \left(-\tilde{m}_{x, t}\right)=\exp \left(-\exp \left(\tilde{y}_{x, t}\right)\right)
$$

under the assumption that the force of mortality between two consecutive integer ages is a constant.

Let $V_{L}(t)$ be the time- $t$ value of the annuity liability, measured in time- $t_{0}$ dollars. We can express $V_{L}(t)$ as

$$
V_{L}(t)=\mathrm{E}\left(L \mid \mathcal{F}_{t}\right), \quad t \geq t_{0}
$$

where $\mathcal{F}_{t}$ denotes the information up to and including time $t$.

When considering a value hedge over a $\tau$-year horizon, we are especially interested in the value of the 


\begin{tabular}{|rcccccc|}
\hline Cash flow & $\$ 0$ & $\cdots$ & $\$ 0$ & $\$ 1$ & $\$ 1$ & $\cdots$ \\
& + & & $\mid$ & $\mid$ & & \\
Age & $x_{0}$ & $\cdots$ & $x_{0}+T_{L}-1$ & $x_{0}+T_{L}$ & $x_{0}+T_{L}+1$ & $\cdots$ \\
Time & $t_{0}$ & $\cdots$ & $t_{0}+T_{L}-1$ & $t_{0}+T_{L}$ & $t_{0}+T_{L}+1$ & $\cdots$ \\
\hline
\end{tabular}

Figure 1: An illustration of the cash flows associated with the liability being hedged: A $T_{L}$-year deferred whole life annuity-due sold to individuals who are aged $x_{0}$ at time $t_{0}$.

liability at time $t=t_{0}+\tau$ :

$$
V_{L}\left(t_{0}+\tau\right)=\mathrm{E}\left(L \mid \mathcal{F}_{t_{0}+\tau}\right)
$$

To keep the mathematics in Section 4 modest, we assume $\tau<T_{L}$ (i.e., the horizon of the hedge is shorter than the deferment period). It is clear that $V_{L}\left(t_{0}+\tau\right)$ is a random variable until time $t_{0}+\tau$. In particular, as of time $t_{0}$ when the hedge is established, $V_{L}\left(t_{0}+\tau\right)$ is subject to the uncertainty surrounding the path of the random state vectors $\vec{\alpha}_{t_{0}+1}, \ldots, \vec{\alpha}_{t_{0}+\tau}$. Equivalently speaking, $V_{L}\left(t_{0}+\tau\right)$ is subject to the randomness associated with the innovation vectors $\vec{\eta}_{t_{0}+1}, \ldots, \vec{\eta}_{t_{0}+\tau}$, because, according to equation (2.2), we can always express the state vector at time $t_{0}+s$ in terms of the innovation vectors at times $t_{0}+1, \ldots, t_{0}+s$ :

$$
\begin{aligned}
\vec{\alpha}_{t_{0}+s} & =\left(\mathbf{I}+\mathbf{A}+\cdots+\mathbf{A}^{s-1}\right) \vec{c}+\mathbf{A}^{s} \vec{\alpha}_{t_{0}}+\vec{\eta}_{t_{0}+s}^{*}+\mathbf{A} \vec{\eta}_{t_{0}+s-1}^{*}+\cdots+\mathbf{A}^{s-1} \vec{\eta}_{t_{0}+1}^{*} \\
& =\left(\mathbf{I}+\mathbf{A}+\cdots+\mathbf{A}^{s-1}\right) \vec{c}+\mathbf{A}^{s} \vec{\alpha}_{t_{0}}+\mathbf{C} \vec{\eta}_{t_{0}+s}+\mathbf{A} \mathbf{C} \vec{\eta}_{t_{0}+s-1}+\cdots+\mathbf{A}^{s-1} \mathbf{C} \vec{\eta}_{t_{0}+1} \\
& =\left(\sum_{u=1}^{s} \mathbf{A}^{s-u} \vec{c}\right)+\mathbf{A}^{s} \vec{\alpha}_{t_{0}}+\left(\sum_{u=1}^{s} \mathbf{A}^{s-u} \mathbf{C} \vec{\eta}_{t_{0}+u}\right), \quad \text { for } s=1,2, \ldots
\end{aligned}
$$

The linkage between $V_{L}\left(t_{0}+\tau\right)$ and $\vec{\eta}_{t_{0}+1}, \ldots, \vec{\eta}_{t_{0}+\tau}$ forms the foundation of the proposed hedging strategy (Section 4) and efficient simulation method (Section 5).

\subsection{The Hedging Instruments}

As with many previous studies on longevity risk hedging (e.g., Cairns, 2011, 2013; Li and Hardy, 2011; Li and Luo, 2012; Zhou and Li, 2017), we use q-forwards as hedging instruments. A q-forward is a zero-coupon swap that exchanges at maturity an amount proportional to the realized mortality rate at a certain age (the reference age) at the time when the swap matures with an amount proportional to a fixed mortality rate (the forward mortality rate). The exchange of cash flows at maturity is illustrated in Figure 2.

Suppose that the hedge portfolio consists of $m$ q-forwards, all of which are freshly launched at time $t_{0}$. Let $H_{j}$, for $j=1, \ldots, m$, be the payoff of the $j$-th q-forward from the fixed-rate receiver's perspective, discounted to time $t_{0}$ at an interest rate of $r$. We have

$$
H_{j}=e^{-r \times T_{j}}\left(q_{x_{j}, t_{0}+T_{j}}^{f}-\tilde{q}_{x_{j}, t_{0}+T_{j}}\right)
$$

where $x_{j}$ is the reference age, $T_{j}$ is the maturity date, and $q_{x_{j}, t_{0}+T_{j}}^{f}$ represents the forward mortality rate that is fixed at time $t_{0}{ }^{2}$

\footnotetext{
${ }^{2}$ We assume that the floating leg is the underlying death probability instead of the observed (crude) death probability. This assumption is consistent with the fact that the LifeMetrics Index (on which q-forwards in today's
} 


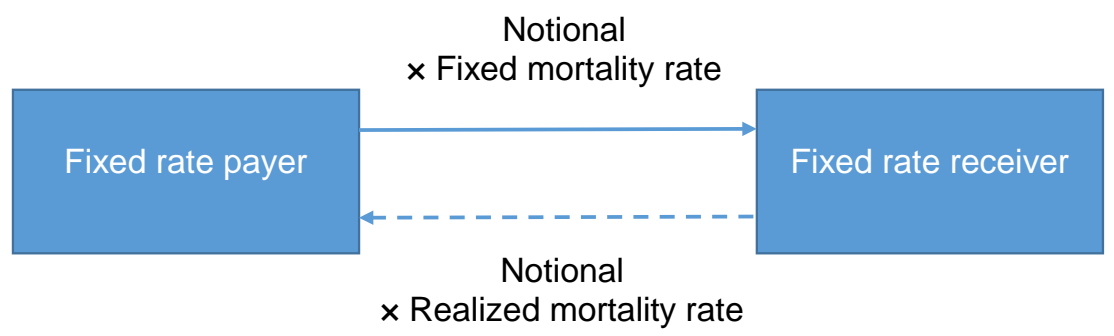

Figure 2: An illustration of the cash flows exchanged between the two counterparties of a q-forward at maturity.

Following Loeys et al. (2007), we assume that the forward mortality rate is determined at time $t_{0}$ as follows:

$$
q_{x_{j}, t_{0}+T_{j}}^{f}=\left(1-T_{j} \times \lambda \times v_{x_{j}}\right) \hat{q}_{x_{j}, t_{0}+T_{j}},
$$

where

- $\hat{q}_{x_{j}, t_{0}+T_{j}}$ represents the best estimate of $q_{x_{j}, t_{0}+T_{j}}$, obtained by setting $\epsilon_{x, t}$ and $\vec{\eta}_{t}$ for all $x$ and $t>t_{0}$ to zero.

- $v_{x_{j}}$ is the estimated volatility of the yearly changes in the death probability at age $x_{j}$, and

- $\lambda$ is the annualized Sharpe ratio.

As pointed out by Loeys et al. (2007), Michaelson and Mulholland (2014) and Graziani (2014), the current longevity market is net short longevity. In other words, there are more participants who would be financially damaged (and thus seeking for protection) than those who would benefit from the unexpected rises in life expectancy. Therefore, $\lambda$ should be positive so that the counterparty taking longevity risk exposures from the hedger is compensated. To the hedger, the cost of hedging is positively related to $\lambda$. In special case when $\lambda=0$, the hedge is costless.

We let

$$
V_{H_{j}}(t)=\mathrm{E}\left(H_{j} \mid \mathcal{F}_{t}\right), \quad t \geq t_{0},
$$

be the time- $t$ value of the $j$-th hedging instrument, measured in time- $t_{0}$ dollars. When constructing a value hedge over a $\tau$-year horizon, of our particular interest is the value of the $j$-th hedging instrument at time $t=t_{0}+\tau$ :

$$
V_{H_{j}}\left(t_{0}+\tau\right)=\mathrm{E}\left(H_{j} \mid \mathcal{F}_{t_{0}+\tau}\right)
$$

For simplicity, we assume $\tau<T_{j}$. It is clear that $V_{H_{j}}\left(t_{0}+\tau\right)$ is a random variable until time $t_{0}+\tau$. Also, similar to $V_{L}\left(t_{0}+\tau\right)$, as of time $t_{0}$ when the hedge is established, $V_{H_{j}}\left(t_{0}+\tau\right)$ is subject to the uncertainty associated with the innovation vectors $\vec{\eta}_{t_{0}+1}, \ldots, \vec{\eta}_{t_{0}+\tau}$.

market can be written) is developed using smoothed death probabilities instead of crude death probabilities. 


\section{Deriving the Optimal Hedging Strategy}

\subsection{The Objective}

We consider a static value hedge over a horizon of $\tau$ years. In particular, our objective is to minimize the Value-at-Risk (at the $100 \gamma \%$ confidence level) of the hedged position $\tau$ years after time $t_{0}$ when the hedge is established.

Let $N_{j}$ be the notional amount of the $j$-th hedging instrument. According to our objective, the notional amounts of the hedging instruments should be optimized as follows:

$$
\min _{N_{1}, \ldots, N_{m}} \operatorname{VaR}_{\gamma}\left(V_{L}\left(t_{0}+\tau\right)-\sum_{j=1}^{m} N_{j} \times V_{H_{j}}\left(t_{0}+\tau\right) \mid \mathcal{F}_{t_{0}}\right),
$$

where $\operatorname{VaR}_{\gamma}(z)$ represents the $100 \gamma$-th percentile of $z$.

However, the optimization problem above is not straightforward to solve. To facilitate the development of an analytical expression of the first order conditions, we find the optimal notional amounts by solving the following optimization problem instead:

$$
\min _{N_{1}, \ldots, N_{m}} \operatorname{VaR}_{\gamma}\left(V_{l}\left(t_{0}+\tau\right)-\sum_{j=1}^{m} N_{j} \times V_{h_{j}}\left(t_{0}+\tau\right) \mid \mathcal{F}_{t_{0}}\right),
$$

where $V_{l}\left(t_{0}+\tau\right)$ and $V_{h_{j}}\left(t_{0}+\tau\right)$ are approximated values of $V_{L}\left(t_{0}+\tau\right)$ and $V_{H_{j}}\left(t_{0}+\tau\right)$, respectively. The approximation is detailed in the next sub-section.

\subsection{The Approximation}

\subsubsection{Setting up the Approximation}

We let $l$ and $h_{j}$ be the first-order Taylor approximations of $L$ and $H_{j}$ around the relevant innovation vectors, respectively. We have

$$
l=\hat{L}+\sum_{s=1}^{\omega-x_{0}}\left(\frac{\partial L}{\partial \vec{\eta}_{t_{0}+s}}\right)^{\prime} \vec{\eta}_{t_{0}+s}
$$

and

$$
h_{j}=\hat{H}_{j}+\sum_{s=1}^{T_{j}}\left(\frac{\partial H_{j}}{\partial \vec{\eta}_{t_{0}+s}}\right)^{\prime} \vec{\eta}_{t_{0}+s}
$$

where $\hat{L}$ and $\hat{H}_{j}$ respectively represent the estimates of $L$ and $H_{j}$ that are computed by setting $\vec{\eta}_{t_{0}+s}$ to $\overrightarrow{0}$ (the expected value of $\vec{\eta}_{t_{0}+s}$ ) for all $s>0$. All partial derivatives with respect to the innovation vectors are evaluated at the expected values of the innovations.

Then, the time- $\left(t_{0}+\tau\right)$ value of the liability (measured in time- $t_{0}$ dollars) can be approximated as 
follows:

$$
\begin{aligned}
V_{L}\left(t_{0}+\tau\right) & \approx V_{l}\left(t_{0}+\tau\right) \\
& :=\mathrm{E}\left(l \mid \mathcal{F}_{t_{0}+\tau}\right) \\
& =\mathrm{E}\left(\hat{L}+\sum_{s=1}^{\omega-x_{0}}\left(\frac{\partial L}{\partial \vec{\eta}_{t_{0}+s}}\right)^{\prime} \vec{\eta}_{t_{0}+s} \mid \mathcal{F}_{t_{0}+\tau}\right) \\
& =\hat{L}+\sum_{s=1}^{\tau}\left(\frac{\partial L}{\partial \vec{\eta}_{t_{0}+s}}\right)^{\prime} \vec{\eta}_{t_{0}+s} .
\end{aligned}
$$

The last step in the above follows from the fact that given $\mathcal{F}_{t_{0}+\tau}$, the expected value of all innovations beyond time $t_{0}+\tau$ is zero. Likewise, the time- $\left(t_{0}+\tau\right)$ value of the $j$-th hedging instrument (measured in time- $t_{0}$ dollars) can be approximated as follows:

$$
\begin{aligned}
V_{H_{j}}\left(t_{0}+\tau\right) & \approx V_{h_{j}}\left(t_{0}+\tau\right) \\
& :=\mathrm{E}\left(h_{j} \mid \mathcal{F}_{t_{0}+\tau}\right) \\
& =\mathrm{E}\left(\hat{H}_{j}+\sum_{s=1}^{T_{j}}\left(\frac{\partial H_{j}}{\partial \vec{\eta}_{t_{0}+s}}\right)^{\prime} \vec{\eta}_{t_{0}+s} \mid \mathcal{F}_{t_{0}+\tau}\right) \\
& =\hat{H}_{j}+\sum_{s=1}^{\tau}\left(\frac{\partial H_{j}}{\partial \vec{\eta}_{t_{0}+s}}\right)^{\prime} \vec{\eta}_{t_{0}+s} .
\end{aligned}
$$

In the rest of this sub-section, we derive the partial derivatives in equations (4.5) and (4.6), on the basis of a mortality model formulated in the general state-space representation.

\subsubsection{Deriving $V_{l}\left(t_{0}+\tau\right)$}

We now focus on the partial derivatives in the expression for $V_{l}\left(t_{0}+\tau\right)$. First of all, following the definition of $L$ in equation (3.1), we have

$$
\frac{\partial L}{\partial \vec{\eta}_{t_{0}+s}}=\sum_{u=1}^{\omega-x_{0}-T_{L}}\left(e^{-r\left(T_{L}+u-1\right)}\left(\frac{\partial}{\partial \vec{\eta}_{t_{0}+s}}\left(\prod_{i=1}^{T_{L}+u-1} \tilde{p}_{x_{0}+i-1, t_{0}+i}\right)\right)\right) .
$$

The partial derivative in the expression above can be further expanded using the product rule and the chain rule as follows:

$$
\begin{aligned}
\frac{\partial}{\partial \vec{\eta}_{t_{0}+s}}\left(\prod_{i=1}^{T_{L}+u-1} \tilde{p}_{x_{0}+i-1, t_{0}+i}\right) & =\sum_{i=1}^{T_{L}+u-1}\left(\frac{\partial \tilde{p}_{x_{0}+i-1, t_{0}+i}}{\partial \vec{\eta}_{t_{0}+s}} \times \prod_{\substack{j=1 \\
j \neq i}}^{T_{L}+u-1} \tilde{p}_{x_{0}+j-1, t_{0}+j}\right) \\
& =\sum_{i=1}^{T_{L}+u-1}\left(\frac{\partial \tilde{p}_{x_{0}+i-1, t_{0}+i}}{\partial \tilde{y}_{x_{0}+i-1, t_{0}+i}} \times \frac{\partial \tilde{y}_{x_{0}+i-1, t_{0}+i}}{\partial \vec{\eta}_{t_{0}+s}} \times \prod_{\substack{j=1 \\
j \neq i}}^{T_{L}+u-1} \tilde{p}_{x_{0}+j-1, t_{0}+j}\right),
\end{aligned}
$$

which involves the partial derivative of $\tilde{p}_{x_{0}+i-1, t_{0}+i}$ with respect to $\tilde{y}_{x_{0}+i-1, t_{0}+i}$ and the partial derivative of $\tilde{y}_{x_{0}+i-1, t_{0}+i}$ with respect to $\vec{\eta}_{t_{0}+s}$. The former depends on the specification of the observations in the 
model. It follows from equations (3.2) and (3.3) that

$$
\frac{\partial \tilde{p}_{x_{0}+i-1, t_{0}+i}}{\partial \tilde{y}_{x_{0}+i-1, t_{0}+i}}=\left\{\begin{array}{ll}
(-1) \times \tilde{p}_{x_{0}+i-1, t_{0}+i} \times \tilde{q}_{x_{0}+i-1, t_{0}+i}, & \text { if } y_{x, t}=\operatorname{logit}\left(q_{x, t}\right) \\
(-1) \times \tilde{p}_{x_{0}+i-1, t_{0}+i} \times \tilde{m}_{x_{0}+i-1, t_{0}+i}, & \text { if } y_{x, t}=\ln \left(m_{x, t}\right)
\end{array} .\right.
$$

The latter can be derived by first expressing $\tilde{y}_{x_{0}+i-1, t_{0}+i}$ in terms of the parameters in the general statespace mortality model as follows:

$$
\tilde{y}_{x_{0}+i-1, t_{0}+i}=d_{x_{0}+i-1}+\mathbf{B}_{x_{0}+i-1, \cdot} \cdot \vec{\alpha}_{t_{0}+i} .
$$

As shown in equation (3.4), the state vector $\vec{\alpha}_{t_{0}+i}$ can be written in terms of the innovations from time $t_{0}+1$ to $t_{0}+i$, so that $\tilde{y}_{x_{0}+i-1, t_{0}+i}$ can be written as

$$
\begin{aligned}
\tilde{y}_{x_{0}+i-1, t_{0}+i} & =d_{x_{0}+i-1}+\mathbf{B}_{x_{0}+i-1, \cdot}\left(\left(\sum_{u=1}^{i} \mathbf{A}^{i-u} \vec{c}\right)+\mathbf{A}^{i} \vec{\alpha}_{t_{0}}+\left(\sum_{u=1}^{i} \mathbf{A}^{i-u} \mathbf{C} \vec{\eta}_{t_{0}+u}\right)\right) \\
& =\text { constant }+\mathbf{B}_{x_{0}+i-1, \cdot}\left(\sum_{u=1}^{i} \mathbf{A}^{i-u} \mathbf{C} \vec{\eta}_{t_{0}+u}\right),
\end{aligned}
$$

which implies that

$$
\frac{\partial \tilde{y}_{x_{0}+i-1, t_{0}+i}}{\partial \vec{\eta}_{t_{0}+s}}=\frac{\partial\left(\mathbf{B}_{x_{0}+i-1, \cdot} \sum_{u=1}^{i} \mathbf{A}^{i-u} \mathbf{C} \vec{\eta}_{t_{0}+u}\right)}{\partial \vec{\eta}_{t_{0}+s}}=\left\{\begin{array}{ll}
\left(\mathbf{B}_{x_{0}+i-1, \cdot} \mathbf{A}^{i-s} \mathbf{C}\right)^{\prime}, & i \geq s \\
\overrightarrow{0}, & i<s
\end{array},\right.
$$

for $s=1, \ldots, \tau$.

\subsubsection{Deriving $V_{h_{j}}\left(t_{0}+\tau\right)$}

We now focus on the partial derivatives in the expression for $V_{h_{j}}\left(t_{0}+\tau\right)$. Let us first expand the partial derivative of $H_{j}$ with respect to $\vec{\eta}_{t_{0}+s}$ as follows:

$$
\begin{aligned}
\frac{\partial H_{j}}{\partial \vec{\eta}_{t_{0}+s}} & =\frac{\partial}{\partial \vec{\eta}_{t_{0}+s}}\left(e^{-r T_{j}}\left(q_{x_{j}, t_{0}+T_{j}}^{f}-\tilde{q}_{x_{j}, t_{0}+T_{j}}\right)\right) \\
& =\frac{\partial}{\partial \vec{\eta}_{t_{0}+s}}\left(e^{-r T_{j}}\left(q_{x_{j}, t_{0}+T_{j}}^{f}-1+\tilde{p}_{x_{j}, t_{0}+T_{j}}\right)\right) \\
& =e^{-r T_{j}} \frac{\partial \tilde{p}_{x_{j}, t_{0}+T_{j}}}{\partial \vec{\eta}_{t_{0}+s}} \\
& =e^{-r T_{j}} \frac{\partial \tilde{p}_{x_{j}, t_{0}+T_{j}}}{\partial \tilde{y}_{x_{j}, t_{0}+T_{j}}} \frac{\partial \tilde{y}_{x_{j}, t_{0}+T_{j}}}{\partial \vec{\eta}_{t_{0}+s}} .
\end{aligned}
$$

In equation (4.13), the partial derivative of $\tilde{p}_{x_{j}, t_{0}+T_{j}}$ with respect to $\tilde{y}_{x_{j}, t_{0}+T_{j}}$ depends on the specification of the observations. It follows from equations (3.2) and (3.3) that

$$
\frac{\partial \tilde{p}_{x_{j}, t_{0}+T_{j}}}{\partial \tilde{y}_{x_{j}, t_{0}+T_{j}}}= \begin{cases}(-1) \times \tilde{p}_{x_{j}, t_{0}+T_{j}} \times \tilde{q}_{x_{j}, t_{0}+T_{j}}, & \text { if } y_{x, t}=\operatorname{logit}\left(q_{x, t}\right) \\ (-1) \times \tilde{p}_{x_{j}, t_{0}+T_{j}} \times \tilde{m}_{x_{j}, t_{0}+T_{j}}, & \text { if } y_{x, t}=\ln \left(m_{x, t}\right)\end{cases}
$$


Similar to equation (4.11), we can express $\tilde{y}_{x_{j}, t_{0}+T_{j}}$ in terms of the innovations from time $t_{0}+1$ to $t_{0}+T_{j}$. Then, the partial derivative of $\tilde{y}_{x_{j}, t_{0}+T_{j}}$ with respect to $\vec{\eta}_{t_{0}+s}$ can be obtained as follows:

$$
\frac{\partial \tilde{y}_{x_{j}, t_{0}+T_{j}}}{\vec{\eta}_{t_{0}+s}}=\frac{\partial\left(\mathbf{B}_{x_{j},}, \sum_{m=1}^{T_{j}} \mathbf{A}^{T_{j}-m} \mathbf{C} \vec{\eta}_{t_{0}+m}\right)}{\partial \vec{\eta}_{t_{0}+s}}=\left\{\begin{array}{ll}
\left(\mathbf{B}_{x_{j}}, \mathbf{A}^{T_{j}-s} \mathbf{C}\right)^{\prime}, & T_{j} \geq s \\
\overrightarrow{0}, & T_{j}<s
\end{array},\right.
$$

for $s=1, \ldots, \tau$.

\subsection{Partial Derivative Calculations for the Example Models}

We now use the three example models described in Section 2.2 to illustrate the partial derivative calculations involved in the approximations for $V_{l}\left(t_{0}+\tau\right)$ and $V_{h_{j}}\left(t_{0}+\tau\right)$.

\subsubsection{The Cairns-Blake-Dowd Model}

For the Cairns-Blake-Dowd model, we have

$$
\mathbf{A}\left(\begin{array}{ll}
1 & 0 \\
0 & 1
\end{array}\right)
$$

which implies

$$
\mathbf{A}^{n}=\left(\begin{array}{ll}
1 & 0 \\
0 & 1
\end{array}\right), \quad n=1,2, \ldots
$$

Also, we have

$$
\mathbf{B}=\left(\begin{array}{cc}
1 & \left(x_{a}-\bar{x}\right) \\
1 & \left(x_{a}+1-\bar{x}\right) \\
\vdots & \vdots \\
1 & \left(x_{b}-\bar{x}\right)
\end{array}\right),
$$

which gives $\mathbf{B}_{x, \cdot}=\left(\begin{array}{ll}1 & (x-\bar{x})\end{array}\right)$. Finally, we have

$$
\mathbf{C}=\left(\begin{array}{ll}
1 & 0 \\
0 & 1
\end{array}\right)
$$

Let us first consider the liability being hedged. Using the expressions for $\mathbf{A}^{n}, \mathbf{B}_{x}$, and $\mathbf{C}$ and equation (4.12), we immediately obtain

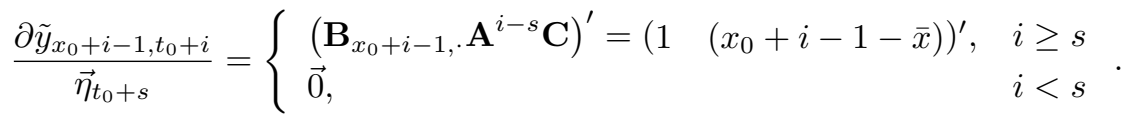

Combining equation (4.16) with equations (4.7) and (4.9) (the case when $y_{x, t}=\operatorname{logit}\left(q_{x, t}\right)$ ), we obtain the 
partial derivative of $L$ with respect to the innovation vector $\vec{\eta}_{t_{0}+s}=\left(\eta_{t_{0}+s}^{(1)}, \eta_{t_{0}+s}^{(2)}\right)^{\prime}$ for $s=1, \ldots, \tau$ :

$$
\frac{\partial L}{\partial \vec{\eta}_{t_{0}+s}}=-\sum_{u=1}^{\omega-x_{0}-T_{L}} e^{-r\left(T_{L}+u\right)}\left(\prod_{i=1}^{T_{L}+u-1} \tilde{p}_{x_{0}+i-1, t_{0}+i}\right) \times\left(\sum_{j=s}^{T_{L}+u-1} \tilde{q}_{x_{0}+j-1, t_{0}+j}\left(1 \quad\left(x_{0}+j-1-\bar{x}\right)\right)^{\prime}\right) .
$$

Elementwise, we have

$$
\frac{\partial L}{\partial \eta_{t_{0}+s}^{(1)}}=-\sum_{u=1}^{\omega-x_{0}-T_{L}} e^{-r\left(T_{L}+u\right)}\left(\prod_{i=1}^{T_{L}+u-1} \tilde{p}_{x_{0}+i-1, t_{0}+i}\right)\left(\sum_{j=s}^{T_{L}+u-1} \tilde{q}_{x_{0}+j-1, t_{0}+j}\right)
$$

and

$$
\frac{\partial L}{\partial \eta_{t_{0}+s}^{(2)}}=-\sum_{u=1}^{\omega-x_{0}-T_{L}} e^{-r\left(T_{L}+u\right)}\left(\prod_{i=1}^{T_{L}+u-1} \tilde{p}_{x_{0}+i-1, t_{0}+i}\right)\left(\sum_{j=s}^{T_{L}+u-1}\left(x_{0}+j-1-\bar{x}\right) \tilde{q}_{x_{0}+j-1, t_{0}+j}\right) .
$$

We then consider the hedging instruments. Using the expressions for $\mathbf{A}^{n}, \mathbf{B}_{x,}$, and $\mathbf{C}$ and equation (4.15), we immediately obtain

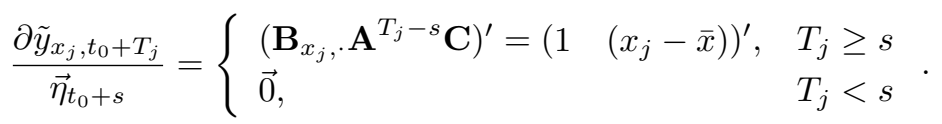

Combining equation (4.17) with equations and (4.13) and (4.14) (the case when $y_{x, t}=\operatorname{logit}\left(q_{x, t}\right)$ ), we obtain the partial derivative of $H_{j}$ with respect to the innovation vector $\vec{\eta}_{t_{0}+s}=\left(\eta_{t_{0}+s}^{(1)}, \eta_{t_{0}+s}^{(2)}\right)^{\prime}$ for $s=1, \ldots, \tau$ :

$$
\frac{\partial H_{j}}{\partial \vec{\eta}_{t_{0}+s}}=-e^{-r T_{j}} \times \tilde{p}_{x_{j}, t_{0}+T_{j}} \times \tilde{q}_{x_{j}, t_{0}+T_{j}} \times\left(1 \quad\left(x_{j}-\bar{x}\right)\right)^{\prime} .
$$

Elementwise, we have

$$
\frac{\partial H_{j}}{\partial \eta_{t_{0}+s}^{(1)}}=-e^{-r T_{j}} \times \tilde{p}_{x_{j}, t_{0}+T_{j}} \times \tilde{q}_{x_{j}, t_{0}+T_{j}}
$$

and

$$
\frac{\partial H_{j}}{\partial \eta_{t_{0}+s}^{(2)}}=-e^{-r T_{j}} \times\left(x_{j}-\bar{x}\right) \times \tilde{p}_{x_{j}, t_{0}+T_{j}} \times \tilde{q}_{x_{j}, t_{0}+T_{j}}
$$

for $s=1, \ldots, \tau$.

\subsubsection{The Lee-Carter Model}

For the Lee-Carter model, we have $\mathbf{A}=1$, which implies $\mathbf{A}^{n}=1$ for any positive integer $n$. Also, as

$$
\mathbf{B}=\left(\begin{array}{llll}
b_{x_{a}} & b_{x_{a}+1} & \cdots & b_{x_{b}}
\end{array}\right)^{\prime},
$$

we have $\mathbf{B}_{x, \cdot}=b_{x}$. Finally, we have $\mathbf{C}=1$. 
Using the expressions for $\mathbf{A}^{n}, \mathbf{B}_{x, \text {. and }} \mathbf{C}$ and equation (4.12), we immediately obtain

$$
\frac{\partial \tilde{y}_{x_{0}+i-1, t_{0}+i}}{\vec{\eta}_{t_{0}+s}}=\left\{\begin{array}{ll}
\left(\mathbf{B}_{x_{0}+i-1,} \cdot \mathbf{A}^{i-s} \mathbf{C}\right)^{\prime}=b_{x_{0}+i-1}, & i \geq s \\
0, & i<s
\end{array} .\right.
$$

Combining equation (4.18) with equations (4.7) and (4.9) (the case when $y_{x, t}=\ln \left(m_{x, t}\right)$ ), we get

$$
\frac{\partial L}{\partial \vec{\eta}_{t_{0}+s}}=\frac{\partial L}{\partial \eta_{t_{0}+s}}=-\sum_{u=1}^{\omega-x_{0}-T_{L}} e^{-r\left(T_{L}+u\right)}\left(\prod_{i=1}^{T_{L}+u-1} \tilde{p}_{x_{0}+i-1, t_{0}+i}\right) \times\left(\sum_{j=s}^{T_{L}+u-1} b_{x_{0}+j-1} \tilde{m}_{x_{0}+j-1, t_{0}+j}\right)
$$

for $s=1, \ldots, \tau$.

Using the expressions for $\mathbf{A}^{n}, \mathbf{B}_{x}$, and $\mathbf{C}$ and equation (4.15), we immediately obtain

$$
\frac{\partial \tilde{y}_{x_{j}, t_{0}+T_{j}}}{\vec{\eta}_{t_{0}+s}}=\left\{\begin{array}{ll}
\left(\mathbf{B}_{x_{j}}, \mathbf{A}^{T_{j}-s} \mathbf{C}\right)^{\prime}=b_{x_{j}}, & T_{j} \geq s \\
0, & T_{j}<s
\end{array} .\right.
$$

Combining equation (4.19) with equations (4.13) and (4.14) (the case when $y_{x, t}=\ln \left(m_{x, t}\right)$ ), we get

$$
\frac{\partial H_{j}}{\partial \vec{\eta}_{t_{0}+s}}=\frac{\partial H_{j}}{\partial \eta_{t_{0}+s}}=-e^{-r T_{j}} \times b_{x_{j}} \times \tilde{m}_{x_{j}, t_{0}+T_{j}} \times \tilde{p}_{x_{j}, t_{0}+T_{j}}
$$

for $s=1, \ldots, \tau$.

\subsubsection{The Renshaw-Haberman Model}

For the Renshaw-Haberman model, we have

$$
\mathbf{A}=\left(\begin{array}{cccccc}
1 & 0 & 0 & \cdots & 0 & 0 \\
0 & \phi_{1} & 0 & \cdots & 0 & 0 \\
0 & 1 & 0 & \cdots & 0 & 0 \\
0 & 0 & 1 & \cdots & 0 & 0 \\
\vdots & \vdots & \vdots & \ddots & \vdots & \vdots \\
0 & 0 & 0 & \cdots & 1 & 0
\end{array}\right)
$$

which implies

$$
\mathbf{A}^{n}=\left(\begin{array}{cccccccc}
1 & 0 & 0 & \cdots & 0 & 0 & \cdots & 0 \\
0 & \phi_{1}^{n} & 0 & \cdots & 0 & 0 & \cdots & 0 \\
0 & \phi_{1}^{n-1} & 0 & \cdots & 0 & 0 & \cdots & 0 \\
\vdots & \vdots & \vdots & & \vdots & \vdots & \vdots & \\
0 & \phi_{1} & 0 & \cdots & 0 & 0 & \cdots & 0 \\
0 & 1 & 0 & \cdots & 0 & 0 & \cdots & 0 \\
0 & 0 & 1 & \cdots & 0 & 0 & \cdots & 0 \\
\vdots & \vdots & \vdots & \ddots & \vdots & \vdots & \vdots & \\
0 & 0 & 0 & \cdots & 1 & 0 & \cdots & 0
\end{array}\right)
$$


for $n=1,2, \ldots$. Also, we have

$$
\mathbf{B}=\left(\begin{array}{cccccc}
b_{x_{a}}^{(k)} & b_{x_{a}}^{(g)} & 0 & \cdots & 0 & 0 \\
b_{x_{a}+1}^{(k)} & 0 & b_{x_{a}+1}^{(g)} & \cdots & 0 & 0 \\
\vdots & \vdots & \vdots & \ddots & \vdots & \vdots \\
b_{x_{b}-1}^{(k)} & 0 & 0 & \cdots & b_{x_{b}-1}^{(g)} & 0 \\
b_{x_{b}}^{(k)} & 0 & 0 & \cdots & 0 & b_{x_{b}}^{(g)}
\end{array}\right)
$$

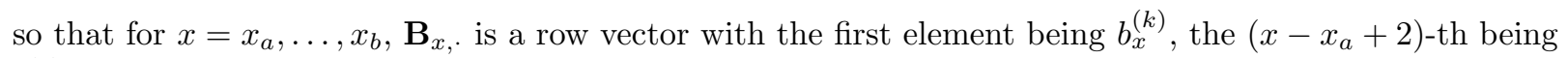
$b_{x}^{(g)}$, and all other elements being zero. Finally, we have

$$
\mathbf{C}=\left(\begin{array}{cc}
1 & 0 \\
0 & 1 \\
0 & 0 \\
\vdots & \vdots \\
0 & 0
\end{array}\right), \text { which gives } \mathbf{A}^{n} \mathbf{C}=\left(\begin{array}{cc}
1 & 0 \\
0 & \phi_{1}^{n} \\
0 & \phi_{1}^{n-1} \\
\vdots & \vdots \\
0 & \phi_{1} \\
0 & 1 \\
0 & 0 \\
\vdots & \vdots \\
0 & 0
\end{array}\right) .
$$

Note that $\mathbf{A}^{n} \mathbf{C}$ is the first two columns of $\mathbf{A}^{n}$, and is devised to relate the hidden states to the nondegenerate portion of $\vec{\eta}_{t}^{*}$ (i.e., $\eta_{t}$ and $\eta_{t-x_{a}}^{(g)}$ ).

Let us first focus on the liability being hedged. Using equations (4.7) and (4.9) (the case when $y_{x, t}=$ $\left.\ln \left(m_{x, t}\right)\right)$, the partial derivative of $L$ with respect to the innovation vector $\vec{\eta}_{t_{0}+s}=\left(\eta_{t_{0}+s}^{(k)}, \eta_{t_{0}-x_{a}+s}^{(g)}\right)^{\prime}$ can be expressed as

$$
\frac{\partial L}{\partial \vec{\eta}_{t_{0}+s}}=-\sum_{u=1}^{\omega-x_{0}-T_{L}} e^{-r\left(T_{L}+u\right)}\left(\prod_{i=1}^{T_{L}+u-1} \tilde{p}_{x_{0}+i-1, t_{0}+i}\right) \times\left(\sum_{j=1}^{T_{L}+u-1} \tilde{m}_{x_{0}+j-1, t_{0}+j} \frac{\partial \tilde{y}_{x_{0}+j-1, t_{0}+j}}{\vec{\eta}_{t_{0}+s}}\right),
$$

for $s=1, \ldots, \tau$, where $\frac{\partial \tilde{y}_{x_{0}+j-1, t_{0}+j}}{\vec{\eta}_{t_{0}+s}}$ can be computed readily using equation (4.12) and the expressions for $\mathbf{B}_{x}$, and $\mathbf{A}^{n} \mathbf{C}$. Elementwise, the partial derivative of $L$ with respect to $\eta_{t_{0}+s}^{(k)}$ is given by

$$
\frac{\partial L}{\partial \eta_{t_{0}+s}^{(k)}}=-\sum_{u=1}^{\omega-x_{0}-T_{L}} e^{-r\left(T_{L}+u\right)}\left(\prod_{i=1}^{T_{L}+u-1} \tilde{p}_{x_{0}+i-1, t_{0}+i}\right) \times\left(\sum_{j=s}^{T_{L}+u-1} b_{x_{0}+j-1} \tilde{m}_{x_{0}+j-1, t_{0}+j}\right)
$$

for $s=1, \ldots, \tau$.

The partial derivative of $L$ with respect to $\eta_{t_{0}-x_{a}+s}^{(g)}$ merits more explanations. Note that the liability being hedged is linked to $g_{t_{0}-x_{0}+1}$ (the cohort effect for year-of-birth $\left(t_{0}-x_{0}+1\right)$ ), which in turn depends on $\eta_{t_{0}-x_{0}+1}^{(g)}, \eta_{t_{0}-x_{0}}^{(g)}, \eta_{t_{0}-x_{0}-1}^{(g)}, \ldots$ (only). As a consequence, if $t_{0}-x_{a}+s>t_{0}-x_{0}+1$, then the partial derivative of $L$ with respect to $\eta_{t_{0}-x_{a}+s}^{(g)}$ is simply zero. Using some simple matrix algebra, it can be shown 
that if $t_{0}-x_{a}+s \leq t_{0}-x_{0}+1$, then the partial derivative of $L$ with respect to $\eta_{t_{0}-x_{a}+s}^{(g)}$ is given by

$$
\frac{\partial L}{\partial \eta_{t_{0}-x_{a}+s}^{(g)}}=-\phi_{1}^{x_{a}-x_{0}+1-s} \sum_{u=1}^{\omega-x_{0}-T_{L}} e^{-r\left(T_{L}+u\right)}\left(\prod_{i=1}^{T_{L}+u-1} \tilde{p}_{x_{0}+i-1, t_{0}+i}\right) \times\left(\sum_{j=1}^{T_{L}+u-1} b_{x_{0}+j-1}^{(g)} \tilde{m}_{x_{0}+j-1, t_{0}+j}\right),
$$

for $s=1, \ldots, \tau$.

We then turn to the hedging instruments. Using equations (4.13) and (4.14) (the case when $y_{x, t}=$ $\left.\ln \left(m_{x, t}\right)\right)$, the partial derivative of $H_{j}$ with respect to the innovation vector $\vec{\eta}_{t_{0}+s}=\left(\eta_{t_{0}+s}^{(k)}, \eta_{t_{0}-x_{a}+s}^{(g)}\right)^{\prime}$ can be expressed as

$$
\frac{\partial H_{j}}{\partial \vec{\eta}_{t_{0}+s}}=-e^{-r T_{j}} \times \tilde{m}_{x_{j}, t_{0}+T_{j}} \times \tilde{p}_{x_{j}, t_{0}+T_{j}} \times \frac{\partial \tilde{y}_{x_{j}, t_{0}+T_{j}}}{\vec{\eta}_{t_{0}+s}}
$$

for $s=1, \ldots, \tau$, where $\frac{\partial \tilde{y}_{x_{j}, t_{0}+T_{j}}}{\vec{\eta}_{t_{0}+s}}$ can be computed readily using equation (4.15) and the expressions for $\mathbf{B}_{x, \text { and }} \mathbf{A}^{n} \mathbf{C}$. Elementwise, we have

$$
\frac{\partial H_{j}}{\partial \eta_{t_{0}+s}^{(k)}}=-e^{-r T_{j}} \times \tilde{m}_{x_{j}, t_{0}+T_{j}} \times \tilde{p}_{x_{j}, t_{0}+T_{j}} \times b_{x_{j}}
$$

and

$$
\frac{\partial H_{j}}{\partial \eta_{t_{0}-x_{a}+s}^{(g)}}= \begin{cases}0, & \left(t_{0}+T_{j}-x_{j}\right)<\left(t_{0}-x_{a}+s\right) \\ -e^{-r T_{j}} \times \tilde{m}_{x_{j}, t_{0}+T_{j}} \times \tilde{p}_{x_{j}, t_{0}+T_{j}} \times b_{x_{j}}^{(g)} \times \phi_{1}^{x_{a}-x_{j}+T_{j}-s}, & \left(t_{0}+T_{j}-x_{j}\right) \geq\left(t_{0}-x_{a}+s\right)\end{cases}
$$

for $s=1, \ldots, \tau$.

\subsection{Solving the Optimization Problem}

Having explained how $V_{l}\left(t_{0}+\tau\right)$ and $V_{h_{j}}\left(t_{0}+\tau\right)$ are computed, we now proceed to solving the optimization problem specified in expression (4.2).

We first consider the case when the innovation vectors in the mortality model are assumed to be (multivariate) normally distributed (an assumption that is typically made in previous studies of stochastic mortality modeling). We then extend our results to the family of multivariate stable elliptical distributions, and finally to a multivariate distribution with both skewness and excess kurtosis. This sub-section is concluded with a remark on the relationship between Value-at-Risk minimization and variance minimization.

\subsubsection{Innovations Following a Multivariate Normal Distribution}

Let us suppose that the innovation vectors are independently and identically distributed, following a multivariate normal distribution with a zero mean vector and a variance-covariance matrix of $\mathbf{Q}$. On the basis of equations (4.5) and (4.6), we know that given $\mathcal{F}_{t_{0}}$, the (approximate) hedged position at time 
$t_{0}+\tau$,

$$
\begin{aligned}
V_{l}\left(t_{0}+\tau\right)-\sum_{j=1}^{m} N_{j} \times V_{h_{j}}\left(t_{0}+\tau\right) & =\hat{L}+\sum_{s=1}^{\tau}\left(\frac{\partial L}{\partial \vec{\eta}_{t_{0}+s}}\right)^{\prime} \vec{\eta}_{t_{0}+s}-\sum_{j=1}^{m} N_{j}\left(\hat{H}_{j}+\sum_{s=1}^{\tau}\left(\frac{\partial H_{j}}{\partial \vec{\eta}_{t_{0}+s}}\right)^{\prime} \vec{\eta}_{t_{0}+s}\right) \\
& =\hat{L}-\sum_{j=1}^{m} N_{j} \hat{H}_{j}+\sum_{s=1}^{\tau}\left(\frac{\partial L}{\partial \vec{\eta}_{t_{0}+s}}-N_{j} \frac{\partial H_{j}}{\partial \vec{\eta}_{t_{0}+s}}\right)^{\prime} \vec{\eta}_{t_{0}+s}
\end{aligned}
$$

follows a (univariate) normal distribution with a mean of

$$
\mathcal{M}=\hat{L}-\sum_{j=1}^{m} N_{j} \times \hat{H}_{j}
$$

and a variance of

$$
\mathcal{S}=\sum_{s=1}^{\tau}\left(\frac{\partial L}{\partial \vec{\eta}_{t_{0}+s}}-\sum_{j=1}^{m} N_{j} \times \frac{\partial H_{j}}{\partial \vec{\eta}_{t_{0}+s}}\right)^{\prime} \mathbf{Q}\left(\frac{\partial L}{\partial \vec{\eta}_{t_{0}+s}}-\sum_{j=1}^{m} N_{j} \times \frac{\partial H_{j}}{\partial \vec{\eta}_{t_{0}+s}}\right)
$$

As a shorthand, we use $\pi_{\gamma}$ to denote the Value-at-Risk over a horizon of $\tau$ years at the $100 \gamma \%$ confidence level. By definition,

$$
\operatorname{Pr}\left(V_{l}\left(t_{0}+\tau\right)-\sum_{j=1}^{m} N_{j} \times V_{h_{j}}\left(t_{0}+\tau\right) \leq \pi_{\gamma} \mid \mathcal{F}_{t_{0}}\right)=\gamma .
$$

Rearranging, we have

$$
\operatorname{Pr}\left(\frac{V_{l}\left(t_{0}+\tau\right)-\sum_{j=1}^{m} N_{j} \times V_{h_{j}}\left(t_{0}+\tau\right)-\mathcal{M}}{\sqrt{\mathcal{S}}} \leq \frac{\pi_{\gamma}-\mathcal{M}}{\sqrt{\mathcal{S}}} \mid \mathcal{F}_{t_{0}}\right)=\gamma,
$$

which gives

$$
\pi_{\gamma}=\mathcal{M}+\sqrt{\mathcal{S}} \cdot \Phi^{-1}(\gamma)
$$

where $\Phi$ represents the standard normal distribution function.

To derive the optimal notional amounts, we take partial derivative of $\pi_{\gamma}$ with respect to $N_{1}, \ldots, N_{m}$ :

$$
\frac{\partial \pi_{\gamma}}{\partial N_{i}}=-\hat{H}_{i}-\frac{\Phi^{-1}(\gamma) \cdot \sum_{s=1}^{\tau}\left(\frac{\partial H_{i}}{\partial \vec{\eta}_{t_{0}+s}}\right)^{\prime} \mathbf{Q}\left(\frac{\partial L}{\partial \vec{\eta}_{t_{0}+s}}-\sum_{j=1}^{m} N_{j} \frac{\partial H_{j}}{\partial \vec{\eta}_{t_{0}+s}}\right)}{\sqrt{\sum_{s=1}^{\tau}\left(\frac{\partial L}{\partial \vec{\eta}_{t_{0}+s}}-\sum_{j=1}^{m} N_{j} \frac{\partial H_{j}}{\partial \vec{\eta}_{t_{0}+s}}\right)^{\prime} \mathbf{Q}\left(\frac{\partial L}{\partial \vec{\eta}_{t_{0}+s}}-\sum_{j=1}^{m} N_{j} \frac{\partial H_{j}}{\partial \vec{\eta}_{t_{0}+s}}\right)}}, \quad i=1, \ldots, m .
$$


The optimal notional amounts $\hat{N}_{1}, \ldots, \hat{N}_{m}$ are calculated by solving the first order conditions,

$$
{\frac{\partial \pi_{\gamma}}{\partial N_{i} N_{i}=\hat{N}_{i}}}=0, \quad i=1, \ldots, m
$$

and verifying the second order conditions.

The intuitions behind the solution can be seen readily when we consider $m=1$ hedging instrument. When $m=1$, the first-order condition boils down to a quadratic equation. The root that satisfies the second-order condition

$$
{\frac{\partial^{2} \pi_{\gamma}}{\partial\left(N_{i}\right)^{2}}}_{\mid N_{1}=\hat{N}_{1}}>0
$$

is

$$
\hat{N}_{1}=\frac{-b-\sqrt{b^{2}-4 a c}}{2 a}
$$

where

$$
\begin{aligned}
& a=\left(\Phi^{-1}(\gamma) \mathcal{A}_{1}\right)^{2}-\left(\hat{H}_{1}\right)^{2} \mathcal{A}_{1}, \\
& b=2\left(-\left(\Phi^{-1}(\gamma)\right)^{2} \mathcal{A}_{1}+\left(\hat{H}_{1}\right)^{2}\right) \mathcal{A}_{2}
\end{aligned}
$$

and

$$
c=\left(\Phi^{-1}(\gamma)\right)^{2}\left(\mathcal{A}_{2}\right)^{2}-\left(\hat{H}_{1}\right)^{2} \mathcal{A}_{3},
$$

and $\mathcal{A}_{1}, \mathcal{A}_{2}$ and $\mathcal{A}_{3}$ are defined by

$$
\begin{aligned}
& \mathcal{A}_{1}=\sum_{s=1}^{\tau}\left(\frac{\partial H_{1}}{\partial \vec{\eta}_{t_{0}+s}}\right)^{\prime} \mathbf{Q}\left(\frac{\partial H_{1}}{\partial \vec{\eta}_{t_{0}+s}}\right), \\
& \mathcal{A}_{2}=\sum_{s=1}^{\tau}\left(\frac{\partial H_{1}}{\partial \vec{\eta}_{t_{0}+s}}\right)^{\prime} \mathbf{Q}\left(\frac{\partial L}{\partial \vec{\eta}_{t_{0}+s}}\right)
\end{aligned}
$$

and

$$
\mathcal{A}_{3}=\sum_{s=1}^{\tau}\left(\frac{\partial L}{\partial \vec{\eta}_{t_{0}+s}}\right)^{\prime} \mathbf{Q}\left(\frac{\partial L}{\partial \vec{\eta}_{t_{0}+s}}\right)
$$

respectively.

It is noteworthy that $\hat{N}_{1}$ exists if and only if $b^{2} \geq 4 a c$. It can be shown that

$$
b^{2}-4 a c=4 \mathcal{A}_{1} \mathcal{A}_{3}\left(\frac{\mathcal{A}_{2}^{2}}{\mathcal{A}_{1} \mathcal{A}_{3}}-1\right)\left(\hat{H}_{1}\right)^{2}\left(\left(\hat{H}_{1}\right)^{2}-\left(\Phi^{-1}(\gamma)\right)^{2} \mathcal{A}_{1}\right) \text {. }
$$

Both $\mathcal{A}_{1}$ and $\mathcal{A}_{3}$ are positive, as they respectively represent the (approximate) variances of $H_{1}$ and $L$. Also, it follows from the interpretations of $\mathcal{A}_{1}, \mathcal{A}_{2}$ and $\mathcal{A}_{3}$ that $\mathcal{A}_{2} /\left(\mathcal{A}_{1} \mathcal{A}_{3}\right)$ represents the square of the (approximate) correlation coefficient between $V_{l}\left(t_{0}+\tau\right)$ and $V_{h_{1}}\left(t_{0}+\tau\right)$, and is thus strictly smaller than 
1. Consequently, a sufficient condition for $b^{2} \geq 4 a c$ is

$$
\left(\hat{H}_{1}\right)^{2} \leq\left(\Phi^{-1}(\gamma)\right)^{2} \mathcal{A}_{1}
$$

which means $\left(\hat{H}_{1}\right)^{2}$ has to be sufficiently small. On the basis of the pricing formula specified in equation (3.5) and (3.6), we have

$$
\hat{H}_{j}=e^{-r T_{j}}\left(\left(1-T_{j} \times \lambda \times v_{x_{j}}\right) \hat{q}_{x_{j}, t_{0}+T_{j}}-\hat{q}_{x_{j}, t_{0}+T_{j}}\right)=-\left(T_{j} \times \lambda \times v_{x_{j}}\right) \hat{q}_{x_{j}, t_{0}+T_{j}} .
$$

Condition (4.26) therefore means that the Sharpe ratio demanded by the q-forward's counterparty cannot be too large. This condition is studied more deeply in Section 6.4 where we examine the sensitivity of the hedging results to the assumed value of $\lambda$.

\subsubsection{Innovations Following a Multivariate Stable Elliptical Distribution}

We now extend the optimization result to the family of multivariate stable elliptical distributions, to which many symmetric distributions including multivariate normal and Student's $t$ belong. A random vector $\vec{X}$ with a dimension of $d$ is said to follow a stable elliptical distribution with a location parameter of $\vec{\mu}$ and a scale parameter of $\mathbf{V}$ if its characteristic function satisfies

$$
\phi_{\vec{X}}(\vec{t})=\mathrm{E}\left(e^{i \vec{t} \vec{X}}\right)=e^{i \vec{t} \vec{\mu}} e^{-|\vec{t} \mathbf{V} \vec{t}|^{\psi / 2}}, \quad \vec{t} \in \mathbb{R}^{d}
$$

where $\psi$ is a parameter that determines the type of the elliptical distribution. When $\psi=2$, the distribution of $\vec{X}$ is multivariate normal.

The following properties of stable multivariate elliptical distribution enable us to obtain an analytical expression for the first-order condition of the optimization problem specified in (4.2):

1. If $\vec{X}$ is a $d$-dimensional random vector which follows a certain type of stable elliptical distribution, then any linear combination $\vec{L}^{\prime} \vec{X}$ of the elements in $\vec{X}$, where $L=\left(l_{1}, l_{2}, \ldots, l_{d}\right)^{\prime}$ is a vector of real constants, also follows (the univariate version of) the same type of stable elliptical distribution. This property can be verified readily by noting that the characteristic function of $\vec{L}^{\prime} \vec{X}$,

$$
\begin{aligned}
\phi_{\mathbf{L} \vec{X}}(\vec{t}) & =\mathrm{E}\left(e^{i \vec{t}^{\prime} \mathbf{L} \vec{X}}\right)=\mathrm{E}\left(e^{i\left(\mathbf{L}^{\prime} \vec{t}\right)^{\prime} \vec{X}}\right)=\phi_{\vec{X}}\left(\mathbf{L}^{\prime} \vec{t}\right) \\
& =e^{i\left(\mathbf{L}^{\prime} \vec{t}\right)^{\prime} \vec{\mu}} e^{-\left|\left(\mathbf{L}^{\prime} \vec{t}\right)^{\prime} \mathbf{V}\left(\mathbf{L}^{\prime} \vec{t}\right)\right|^{\psi / 2}}=e^{i \overrightarrow{t^{\prime}(\mathbf{L} \vec{\mu})} e^{-\left|\vec{t}^{\prime}\left(\mathbf{L} \mathbf{V} \mathbf{L}^{\prime}\right) \vec{t}\right|^{\psi / 2}}},
\end{aligned}
$$

is the characteristic function of the same type of stable elliptical distribution with a location parameter of $\mathbf{L} \vec{\mu}$ and a scale parameter of $\mathbf{L} \mathbf{V} \mathbf{L}^{\prime}$.

2. Suppose that $X_{1}, \ldots, X_{n}$ are $n$ independent random variables, which follow stable elliptical distributions with the same value of $\psi$, but different location and scale parameters. Then, $\sum_{j=1}^{n} X_{j}$ also follows the same type of stable elliptical distribution. This property can be verified by noting that 
the characteristic function of $\sum_{j=1}^{n} X_{j}$

$$
\begin{aligned}
\phi_{\sum_{j=1}^{n} X_{j}}(t) & =\mathrm{E}\left(e^{i t \sum_{j=1}^{n} X_{j}}\right)=\prod_{j=1}^{n} \mathrm{E}\left(e^{i t X_{j}}\right) \\
& =\prod_{j=1}^{n} e^{i t \mu_{j}} e^{-\left|v_{j}\right|^{\psi / 2} t^{\psi}}=e^{i t \sum_{j=1}^{n} \mu_{j}} e^{-\left(\sum_{j=1}^{n}\left|v_{j}\right|^{\psi / 2} t^{\psi}\right)},
\end{aligned}
$$

where $\mu_{i}$ and $v_{i}$ represent the location and scale parameters for $X_{i}$, respectively, is the characteristic function of the same type of stable elliptical distribution with a location parameter of $\sum_{j=1}^{n} \mu_{j}$ and a scale parameter of $\left(\sum_{j=1}^{n}\left|v_{j}\right|^{\psi / 2}\right)^{2 / \psi}$.

Let us now assume that the innovation vectors follow a certain multivariate stable elliptical distribution with a zero mean vector and a constant variance-covariance matrix $\mathbf{Q}$. The first property guarantees that

$$
\left(\frac{\partial L}{\partial \vec{\eta}_{t_{0}+s}}-N_{j} \frac{\partial H_{j}}{\partial \vec{\eta}_{t_{0}+s}}\right)^{\prime} \vec{\eta}_{t_{0}+s}, \quad s=1, \ldots, \tau
$$

follows (the univariate version of) the same type of stable elliptical distribution. Furthermore, using the second property and the assumption that the innovation vectors are not serially correlated, we know that

$$
\sum_{s=1}^{\tau}\left(\frac{\partial L}{\partial \vec{\eta}_{t_{0}+s}}-N_{j} \frac{\partial H_{j}}{\partial \vec{\eta}_{t_{0}+s}}\right)^{\prime} \vec{\eta}_{t_{0}+s}
$$

also follows (the univariate version of) the same type of stable elliptical distribution. As a consequence, the (approximate) hedged position specified in equation (4.20) follows (the univariate version of) the same type of elliptical distribution with a mean of $\mathcal{M}$ and a variance of $\mathcal{S}$, which are specified in equations (4.21) and (4.22), respectively. Having known the distribution of the hedged position, the first-order condition can be derived using a procedure similar to that described in equations (4.23) to (4.25).

To illustrate, let us suppose that the innovation vectors follow a multivariate Student's $t$ distribution with a zero mean vector, a variance-covariance matrix of $\mathbf{Q}$ and $\nu$ degrees of freedom. Under this distributional assumption, the Value-at-Risk of the (approximate) hedged position over a horizon of $\tau$ years at the $100 \gamma \%$ confidence level is given by

$$
\pi_{\gamma}=\mathcal{M}+\sqrt{\mathcal{S}} \cdot t_{\nu}^{-1}(\gamma)
$$

where $t_{\nu}$ is the distribution function of a standardized (univariate) Student's $t$ distribution with $\nu$ degrees of freedom. The first-order condition to the optimization problem then becomes:

$$
\frac{\partial \pi_{\gamma}}{\partial N_{i}}=-\hat{H}_{i}-\frac{t_{\nu}^{-1}(\gamma) \cdot \sum_{s=1}^{\tau}\left(\frac{\partial H_{i}}{\partial \vec{\eta}_{t_{0}+s}}\right)^{\prime} \mathbf{Q}\left(\frac{\partial L}{\partial \vec{\eta}_{t_{0}+s}}-\sum_{j=1}^{m} N_{j} \frac{\partial H_{j}}{\partial \vec{\eta}_{t_{0}+s}}\right)}{\sqrt{\sum_{s=1}^{\tau}\left(\frac{\partial L}{\partial \vec{\eta}_{t_{0}+s}}-\sum_{j=1}^{m} N_{j} \frac{\partial H_{j}}{\partial \vec{\eta}_{t_{0}+s}}\right)^{\prime} \mathbf{Q}\left(\frac{\partial L}{\partial \vec{\eta}_{t_{0}+s}}-\sum_{j=1}^{m} N_{j} \frac{\partial H_{j}}{\partial \vec{\eta}_{t_{0}+s}}\right)}}=0,
$$

for $i=1, \ldots, m$.

The optimization result may be pushed further to distributions that belong to the family of multivariate elliptical distributions (which encompasses all stable multivariate elliptical distributions). However, not 
all distributions in this family satisfy the second property above; that is, if $X_{1}, \ldots, X_{n}$ follow elliptical distributions (beyond the family of stable elliptical distributions) with the same value of $\psi$, then $\sum_{j=1}^{n} X_{j}$ does not necessarily follow an elliptical distribution with the same value of $\psi$. This limitation is not of a concern if the hedger's objective is to minimize the Value-at-Risk over a horizon of $\tau=1$ year (a risk measure that is particularly relevant to Solvency II capital requirements).

\subsubsection{Innovations following a Multivariate Distribution with Skewness and Excess Kurtosis}

If the innovations of the assumed stochastic mortality model exhibit both skewness and excess kurtosis, we recommend modeling the innovation vectors using the multivariate skew- $t$ distribution developed by Azzalini and Capitanio (2003). ${ }^{3}$

We use

$$
\vec{Y} \sim \operatorname{St}_{d}(\vec{\xi}, \Omega, \vec{\zeta}, \nu)
$$

to denote a $d$-dimensional random vector $\vec{Y}$ which follows Azzalini and Capitanio's (2003) multivariate skew- $t$ distribution with a location parameter of $\vec{\xi}$, a dispersion parameter of $\Omega$, a skewness parameter of $\vec{\zeta}$ and $\nu$ degrees of freedom. The density function of $\vec{Y}$ is given by

$$
f_{\vec{Y}}(\vec{y})=2 t_{d}(\vec{y} ; \nu) T_{1}\left(\vec{\zeta}^{\prime} \Lambda^{-1}(\vec{y}-\vec{\xi})\left(\frac{\nu+d}{\delta_{\vec{y}}+\nu}\right)^{1 / 2} ; \nu+d\right) .
$$

In the above expression, $\vec{\xi}$ and $\vec{\zeta}$ are $d$-by-1 constant vectors, $\Omega$ is a full rank symmetric $d$-by- $d$ matrix, $\nu$ is a constant scalar,

$$
\Lambda=\operatorname{diag}\left(\sigma_{1,1}, \ldots, \sigma_{d, d}\right)^{1 / 2}
$$

where $\sigma_{i, i}$ represents the $i$-th element in $\Omega, T_{1}(\cdot ; \nu+d)$ is the distribution function of the univariate Student's $t$ with $\nu+d$ degrees of freedom,

$$
\delta_{\vec{y}}=(\vec{y}-\vec{\xi})^{\prime} \Omega^{-1}(\vec{y}-\vec{\xi})
$$

is the quadratic form of the standardization of $\vec{y}$, and

$$
t_{d}(\vec{y} ; \nu)=\frac{\Gamma\{(\nu+d) / 2\}}{|\Omega|^{1 / 2}(\pi \nu)^{d / 2} \Gamma(\nu / 2)}\left(1+\frac{\delta_{\vec{y}}}{\nu}\right)^{-(\nu+d) / 2},
$$

where $\Gamma$ denotes the gamma function, is the density function of a multivariate $t$ distribution with $\nu$ degrees of freedom.

It follows from the results of Azzalini and Capitanio (2003, Section 4.2.3) that any linear combination of the components of $\vec{Y}$ also follows (the univariate version of) the same skew- $t$ distribution. In particular,

$$
\vec{L}^{\prime} \vec{Y} \sim \mathrm{St}_{1}\left(\vec{L}^{\prime} \vec{\xi}, \vec{L} \Omega \vec{L}^{\prime}, \zeta^{*}, \nu\right)
$$

\footnotetext{
${ }^{3}$ Skew- $t$ distributions are often applied to financial data with skewness and excess kurtosis (see. e.g., Theodossiou, 1998).
} 
where $\zeta^{*}$ is the skewness parameter for which an explicit expression is given by Azzalini and Capitanio (1999).

Suppose that the innovation vectors in the assumed mortality model follow Azzalini and Capitanio's (2003) multivariate skew- $t$ distribution with a zero mean vector, a variance-covariance matrix of $\mathbf{Q}$, a certain skewness parameter and $\nu$ degrees of freedom. The property specified in (4.30) ensures that

$$
\left(\frac{\partial L}{\partial \vec{\eta}_{t_{0}+1}}-N_{j} \frac{\partial H_{j}}{\partial \vec{\eta}_{t_{0}+1}}\right)^{\prime} \vec{\eta}_{t_{0}+1}
$$

follows $\mathrm{St}_{1}$ with a zero mean and a variance of

$$
\left(\frac{\partial L}{\partial \vec{\eta}_{t_{0}+1}}-\sum_{j=1}^{m} N_{j} \times \frac{\partial H_{j}}{\partial \vec{\eta}_{t_{0}+1}}\right)^{\prime} \mathbf{Q}\left(\frac{\partial L}{\partial \vec{\eta}_{t_{0}+1}}-\sum_{j=1}^{m} N_{j} \times \frac{\partial H_{j}}{\partial \vec{\eta}_{t_{0}+1}}\right)
$$

a skewness parameter that can be computed using the explicit expression given by Azzalini and Capitanio (1999), and $\nu$ degrees of freedom. As a result, when considering a horizon of $\tau=1$ year, the (approximate) hedged position specified by equation (4.20) follows $\mathrm{St}_{1}$ with tractable parameters. The first-order condition for the optimization problem can thus be derived using a procedure similar to that described in equations $(4.23)$ to $(4.25)$.

\subsubsection{Value-at-Risk Minimization vs. Variance Minimization}

If our objective is instead minimizing the variance of the (approximate) hedged position (equation (4.20)) given $\mathcal{F}_{t_{0}}$, then the first-order condition can be obtained by setting the first partial derivatives of $\mathcal{S}$ (specified in equation (4.22)) with respect to $N_{1} \ldots, N_{m}$ to zero. The solution to the first order condition is given by

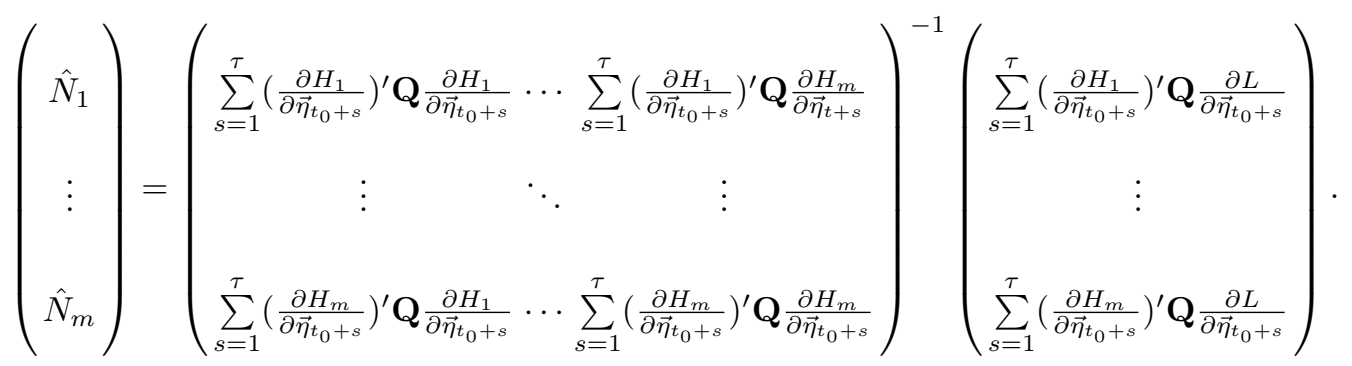

This solution can be shown to be consistent with that obtained by Liu and Li (2017a), who considered variance minimization based on the linkage of $L$ and $H_{j}$ to the state vectors instead of the innovation vectors. Note that equation (4.31) does not depend on the Sharpe ratio $\lambda$ demanded by the counterparty.

It is also interesting to note the relationship between Value-at-Risk minimization and variance minimization when the Sharpe ratio is zero. If $\lambda=0$, then the forward mortality rate $q_{x_{j}, t_{0}+T_{j}}^{f}$ equals $\hat{q}_{x_{j}, t_{0}+T_{j}}$, the best estimate of $q_{x_{j}, t_{0}+T_{j}}$ calculated by switching off all random innovations beyond time $t_{0}$. As such, we have

$$
\hat{H}_{j}=e^{-r T_{j}}\left(\hat{q}_{x_{j}, t_{0}+T_{j}}-\hat{q}_{x_{j}, t_{0}+T_{j}}\right)=0 .
$$

If we assume further that the innovation vectors follow a normal distribution, then the optimal notional amounts obtained by minimizing Value-at-Risk and minimizing variance are identical. The equivalence can 
be seen easily when $m=1$ instrument is used. For $m=1$, when $\hat{H}_{j}=0$, we have

$$
a=\left(\Phi^{-1}(\gamma) \mathcal{A}_{1}\right)^{2}, \quad b=-2\left(\Phi^{-1}(\gamma)\right)^{2} \mathcal{A}_{1} \mathcal{A}_{2} \quad \text { and } \quad c=\left(\Phi^{-1}(\gamma) \mathcal{A}_{2}\right)^{2}
$$

and consequently the notional amount that minimizes the Value-at-Risk over a horizon of $\tau$ years at any confidence level is

$$
\hat{N}_{1}=\frac{-b-\sqrt{b^{2}-4 a c}}{2 a}=\frac{\mathcal{A}_{2}}{\mathcal{A}_{1}}=\frac{\sum_{s=1}^{\tau}\left(\frac{\partial H_{1}}{\partial \vec{\eta}_{t_{0}+s}}\right)^{\prime} \mathbf{Q}\left(\frac{\partial L}{\partial \vec{\eta}_{t_{0}+s}}\right)}{\sum_{s=1}^{\tau}\left(\frac{\partial H_{1}}{\partial \vec{\eta}_{t_{0}+s}}\right)^{\prime} \mathbf{Q}\left(\frac{\partial H_{1}}{\partial \vec{\eta}_{t_{0}+s}}\right)},
$$

which is the same as the expression for $\hat{N}_{1}$ when the objective is to minimize variance over a horizon of $\tau$ years.

The equivalence between Value-at-Risk minimization and variance minimization does not hold if $\lambda$ is not zero and/or the innovation vectors are not normally distributed. That being said, in general situations, the notional amounts that minimize variance are suboptimal if the hedger's actual objective is to minimize Value-at-Risk.

\section{Simulation Methods}

This section focuses on the evaluation of the longevity hedge, that is, the calculation of the Value-at-Risk or variance of the (exact) hedged position

$$
V_{L}\left(t_{0}+\tau\right)-\sum_{j=1}^{m} N_{j} \times V_{H_{j}}\left(t_{0}+\tau\right)
$$

given $\mathcal{F}_{t_{0}}$. We first explain the computational burden entailed in the evaluation, and then propose a method to mitigate the burden.

\subsection{The Potential Computational Burden}

In this paper, we statically hedge the value of an annuity liability at some future time point $\left(t=t_{0}+\tau\right)$. Such a longevity hedge may be evaluated using simulations, but a direct simulation approach is computationally demanding.

To explain, let us suppose that the hedge is evaluated using $M_{1}$ realizations of $V_{L}\left(t_{0}+\tau\right)$ and $V_{H_{j}}\left(t_{0}+\tau\right)$. As explained in Section $3, V_{L}\left(t_{0}+\tau\right)$ and $V_{H_{j}}\left(t_{0}+\tau\right)$ are subject to the uncertainty surrounding the state vectors $\vec{\alpha}_{t_{0}+1}, \ldots, \vec{\alpha}_{t_{0}+\tau}$ from time $t_{0}+1$ to $t_{0}+\tau$. Therefore, in the first step of the evaluation, we need to generate $M_{1}$ sample paths of $\vec{\alpha}_{t_{0}+1}, \ldots, \vec{\alpha}_{t_{0}+\tau}$.

Then, for each of the $M_{1}$ simulated sample paths of the state vectors from time $t_{0}+1$ to $t_{0}+\tau$, we have to calculate the realized values of $V_{L}\left(t_{0}+\tau\right)$ and $V_{H_{j}}\left(t_{0}+\tau\right)$. Because $V_{L}\left(t_{0}+\tau\right)=\mathrm{E}\left(L \mid \mathcal{F}_{t_{0}+\tau}\right)$ and $V_{H_{j}}\left(t_{0}+\tau\right)=\mathrm{E}\left(H_{j} \mid \mathcal{F}_{t_{0}+\tau}\right)$ have no exact analytical form, another set of (say $M_{2}$ ) sample paths of the state vectors beyond time $t_{0}+\tau$ have to be generated to evaluate these expectations.

Further, because of the serial correlations in state vectors, the state vectors beyond time $t_{0}+\tau$ depend 
on the state vectors at time $t_{0}+\tau$ and before. As illustrated in Figure 3, what this means is that we need to generate additional $M_{2}$ sets of sample paths for each one of the $M_{1}$ sets of sample paths. In effect, $M_{1} \times M_{2}$ sets of sample paths are required. Generally speaking, $M_{1}$ and $M_{2}$ are large, say 10,000, and therefore the computation burden is substantial.

\subsection{An Innovation-Based Simulation Procedure}

We now propose a method to save the need for nested simulations. As discussed in Section $3, L$ and $H_{j}$ can be expressed as functions in which the innovation vectors $\vec{\eta}_{t_{0}+s}, s=1,2, \ldots$, are the only random components. Using this fact, in the proposed method we simulate sample paths of the innovations vectors rather than the state vectors. We can then use the generic assumption that the innovation vectors are not serially correlated by design to save computational effort.

Let us explain the proposed method in more detail. According to the relationship specified in equation (3.4), we can regard the innovation vectors $\vec{\eta}_{t_{0}+1}, \ldots, \vec{\eta}_{t_{0}+\tau}$ instead of the state vectors $\vec{\alpha}_{t_{0}+1}, \ldots, \vec{\alpha}_{t_{0}+\tau}$ as the origin of the randomness surrounding $V_{L}\left(t_{0}+\tau\right)$ and $V_{H_{j}}\left(t_{0}+\tau\right)$. Therefore, to evaluate the hedge using $M_{1}$ realizations of $V_{L}\left(t_{0}+\tau\right)$ and $V_{H_{j}}\left(t_{0}+\tau\right)$, we can generate $M_{1}$ sample paths of $\vec{\eta}_{t_{0}+1}, \ldots, \vec{\eta}_{t_{0}+\tau}$ instead.

Still, for each simulated sample paths of $\vec{\eta}_{t_{0}+1}, \ldots, \vec{\eta}_{t_{0}+\tau}$, we have to compute the realized values of $V_{L}\left(t_{0}+\tau\right)=\mathrm{E}\left(L \mid \mathcal{F}_{t_{0}+\tau}\right)$ and $V_{H_{j}}\left(t_{0}+\tau\right)=\mathrm{E}\left(H_{j} \mid \mathcal{F}_{t_{0}+\tau}\right)$. The computation of $V_{L}\left(t_{0}+\tau\right)$ and $V_{H_{j}}\left(t_{0}+\tau\right)$ can be accomplished using $M_{2}$ simulated sample paths of $\vec{\eta}_{t_{0}+\tau+1}, \vec{\eta}_{t_{0}+\tau+2}, \ldots$ Because the innovation vectors possess no serial correlation, the innovation vectors used for valuation at $t=t_{0}+\tau$ do not depend on the realized innovation vectors at $t=t_{0}+1, \ldots, t_{0}+\tau$. Hence, the same set of $M_{2}$ sample paths of $\vec{\eta}_{t_{0}+\tau+1}, \vec{\eta}_{t_{0}+\tau+2}, \ldots$ for valuation purposes can be used for each of the $M_{1}$ sample paths of $\vec{\eta}_{t_{0}+1}, \ldots, \vec{\eta}_{t_{0}+\tau}$, thereby avoiding the need for nested simulations.

The idea is illustrated in Figure 4. For ease of exposition, we assume here that $M_{2}=M_{1}$. As shown in the diagram, $M_{1}$ sample paths of the innovation vectors for $t=t_{0}+1, \ldots, t_{0}+\tau, t_{0}+\tau+1, \ldots, t_{0}+\omega-x_{0}$ are generated. Since the innovation vectors are not serially correlated, $\vec{\eta}_{t_{0}+s}$ for $s>\tau$ does not depend on the realizations of $\vec{\eta}_{t_{0}+s}$ for $s \leq \tau$. As such, we can use the same collection of innovation vectors (the portion enclosed in the rectangle) to calculate $V_{L}\left(t_{0}+\tau\right)$ and $V_{H_{j}}\left(t_{0}+\tau\right)$ for each note $i=1, \ldots, M_{1}$ at time $t_{0}+\tau$. In the entire process of hedge effectiveness evaluation, only $M_{1}$ sample paths of innovation vectors (from time $t_{0}+1$ to $t_{0}+\omega-x_{0}$ ) are needed.

We conclude this section with the following remark. The approximation method presented in Section 4.2 is used only in the derivation of the hedging strategy, but not in the evaluation of the longevity hedge. The procedure proposed in this section expedites simulations by making use of the statistical properties of the innovations but not any approximation.

\section{$6 \quad$ Illustrations}

\subsection{The Assumed Mortality Model}

For the purpose of illustration, we consider the Cairns-Blake-Dowd model (described in Section 2.2.1). The model is fitted to the death and exposure counts from the U.S. male population over an age range of 55 to 


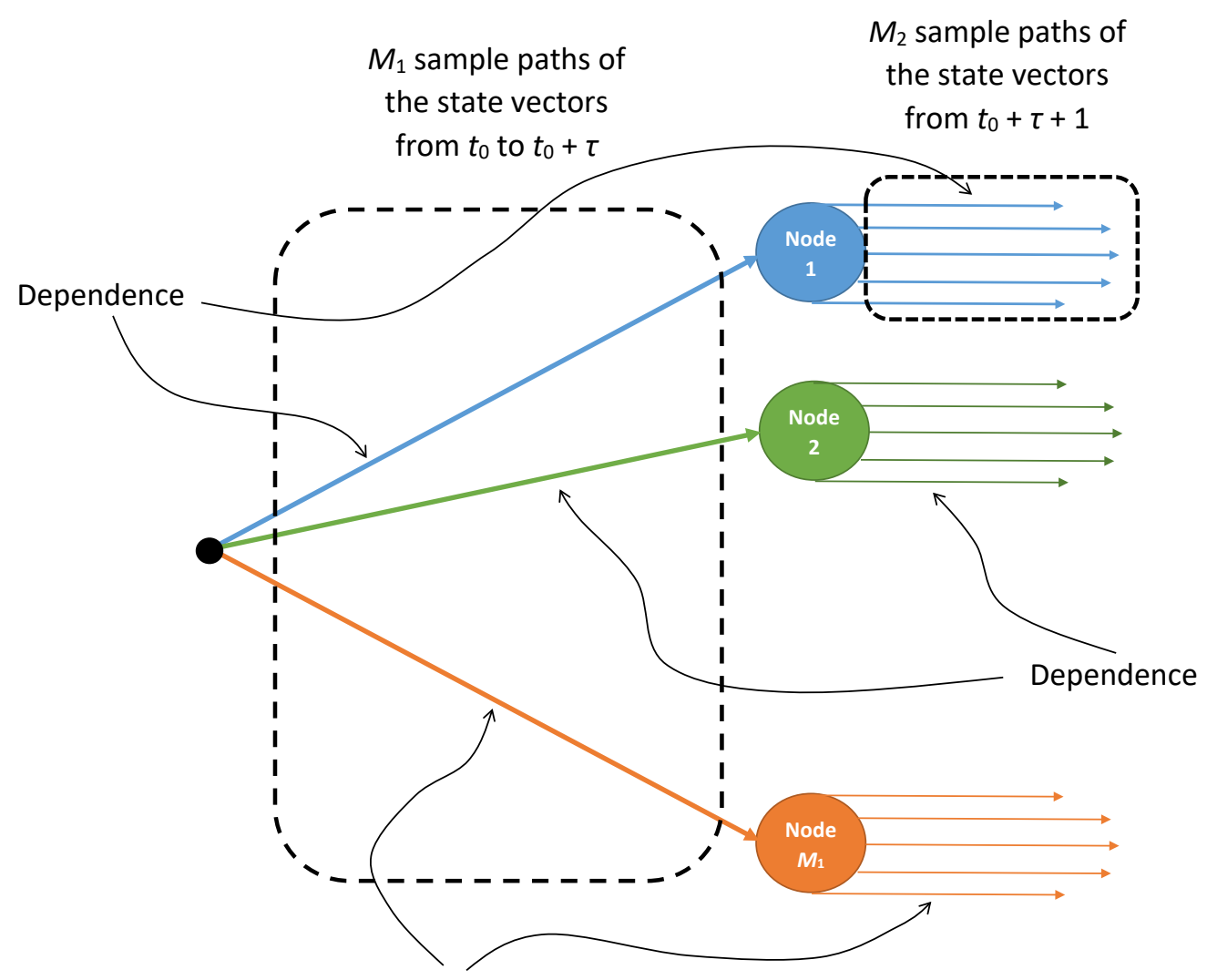

Dependence

Figure 3: An illustration of the computational burden entailed in the evaluation of a value hedge if a direct simulation approach is used.

\begin{tabular}{ccc|ccc}
\cline { 4 - 5 }$\vec{\eta}_{t_{0}+1}(1)$ & $\cdots$ & $\vec{\eta}_{t_{0}+\tau}(1)$ & $\vec{\eta}_{t_{0}+\tau+1}(1)$ & $\cdots$ & $\vec{\eta}_{t_{0}+\omega-x_{0}}(1)$ \\
$\vec{\eta}_{t_{0}+1}(2)$ & $\cdots$ & $\vec{\eta}_{t_{0}+\tau}(2)$ & $\vec{\eta}_{t_{0}+\tau+1}(2)$ & $\cdots$ & $\vec{\eta}_{t_{0}+\omega-x_{0}}(2)$ \\
$\vdots$ & $\vdots$ & $\vdots$ & $\vdots$ & $\vdots$ & $\vdots$ \\
$\vec{\eta}_{t_{0}+1}\left(M_{1}\right)$ & $\cdots$ & $\vec{\eta}_{t_{0}+\tau}\left(M_{1}\right)$ & $\vec{\eta}_{t_{0}+\tau+1}\left(M_{1}\right)$ & $\cdots$ & $\vec{\eta}_{t_{0}+\omega-x_{0}}\left(M_{1}\right)$ \\
\cline { 4 - 6 } & & &
\end{tabular}

Figure 4: An illustration of the innovation-based simulation method. In the diagram, $\vec{\eta}_{u}(i)$ represents the innovation vector at $t=u$ in the $i$-th sample path. The block enclosed by the rectangle is used for computing $V_{L}\left(t_{0}+\tau\right)$ and $V_{H_{j}}\left(t_{0}+\tau\right)$ for each node $i$ at $t=t_{0}+\tau$.

99 and a sample period of 1940 to 2010, using the method of Poisson maximum likelihood. The data are obtained from the Human Mortality Database.

Figure 5 shows the estimates of the hidden states $\vec{\alpha}_{t}=\left(k_{t}^{(1)}, k_{t}^{(2)}\right)^{\prime}$ over the sample period. Parameters $\vec{c}$ and $\mathbf{Q}$ in the transition equation are then estimated using the sample mean and variance-covariance matrix of $\left(\Delta k_{t}^{(1)}, \Delta k_{t}^{(2)}\right)^{\prime}$, where $\Delta$ is the difference operator, over the sample period. The estimates of $\vec{c}$ and $\mathbf{Q}$ are shown in Table 1. 

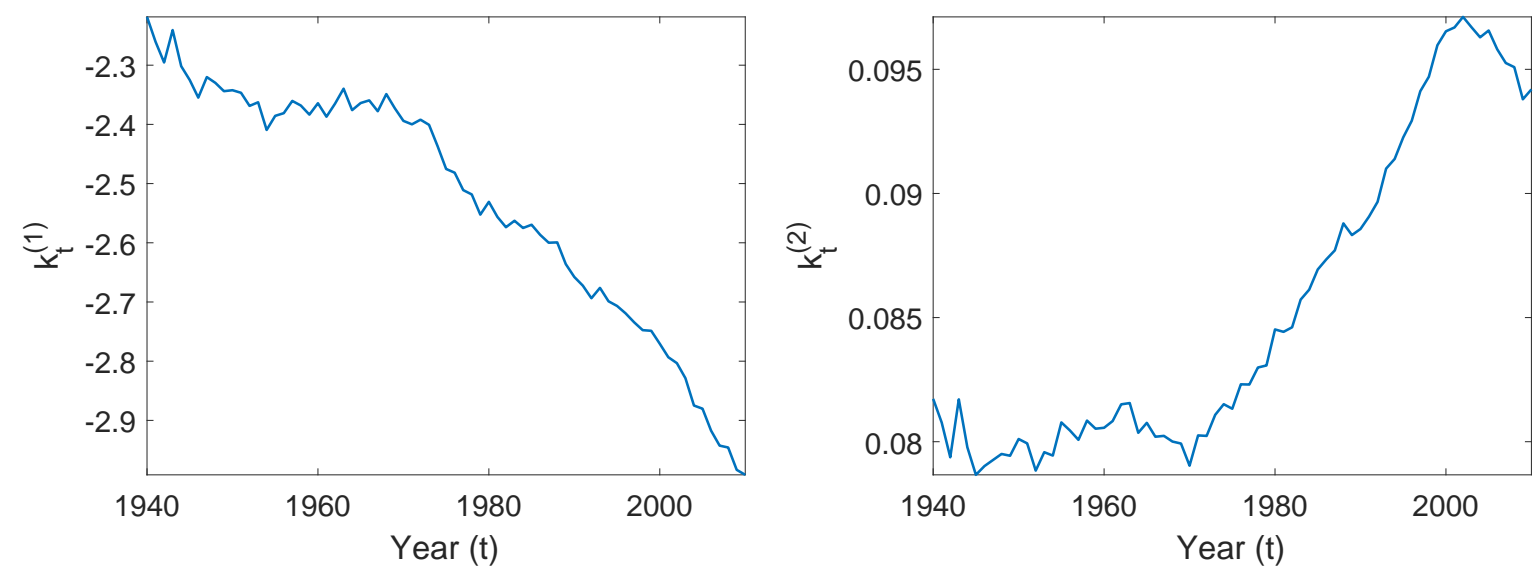

Figure 5: Estimates of the hidden states $\vec{\alpha}_{t}=\left(k_{t}^{(1)}, k_{t}^{(2)}\right)^{\prime}$ in the Cairns-Blake-Dowd model over the sample period of 1940 to 2010 .

\begin{tabular}{c|c}
\hline$\vec{c}$ & $\mathbf{Q}$ \\
\hline$\left(\begin{array}{cc}-0.0111 \\
1.7807 \times 10^{-4}\end{array}\right)$ & $\left(\begin{array}{ll}4.6998 \times 10^{-4} & 9.8220 \times 10^{-6} \\
9.8220 \times 10^{-6} & 5.8294 \times 10^{-7}\end{array}\right)$ \\
\hline
\end{tabular}

Table 1: Estimates of $\vec{c}$ and $\mathbf{Q}$ in the transition equation of the Cairns-Blake-Dowd model.

\subsection{The Baseline Assumptions}

The following assumptions are made in the illustrations.

- The hedge is established at the end of year $2010\left(t_{0}=2010\right)$.

- The liability being hedged is a 10-year deferred $\left(T_{L}=10\right)$ whole life annuity-due sold to individuals aged $x_{0}=55$ at time $t_{0}=2010$.

- The hedge contains one q-forward $(m=1)$.

- The reference age and time-to-maturity of the q-forward used is $x_{1}=65$ and $T_{1}=10$ years, respectively.

- Both the annuity liability and q-forward are linked to the mortality of the U.S. male population.

- The interest rate is $r=0.01$.

- In line with Solvency II, we consider a horizon of one year $(\tau=1)$ and a confidence level of $\gamma=0.995$.

\subsection{Accuracy of the Method in Section 4.2}

In the derivation of the optimal hedging strategy, we have approximated $V_{L}\left(t_{0}+\tau\right)$ and $V_{H_{j}}\left(t_{0}+\tau\right)$ with $V_{l}\left(t_{0}+\tau\right)$ and $V_{h_{j}}\left(t_{0}+\tau\right)$, respectively (see Section 4.2). Before presenting the hedging results, we validate the quality of the approximations.

To perform the validation, we simulate a large number of realizations of $\vec{\eta}_{t_{0}+1}$ (under the assumption that the innovation vectors follow a bivariate normal distribution). Then, for each of the realizations, we calculate both the exact values $\left(V_{L}\left(t_{0}+1\right)\right.$ and $\left.V_{H_{1}}\left(t_{0}+1\right)\right)$ and approximated values $\left(V_{l}\left(t_{0}+1\right)\right.$ and 
$\left.V_{h_{1}}\left(t_{0}+1\right)\right)$ on the basis of the baseline assumptions described in the previous sub-section. The validation results are presented in Figures 6 and 7.

In Figure 6, we compare the empirical distributions of the exact and approximated values. For both the annuity liability and q-forward, the empirical distributions of the exact and approximated values are highly similar to each other, suggesting that the approximation method is very accurate. In Figure 7, we present the scattered plots of $V_{l}\left(t_{0}+1\right)$ against $V_{L}\left(t_{0}+1\right)$ and $V_{h_{1}}\left(t_{0}+1\right)$ against $V_{H_{1}}\left(t_{0}+1\right)$. For each scattered plot, the points lie almost perfectly on the 45 degree line that passes through the origin, offering another confirmation for the accuracy of the approximation method.

The validation is also performed for other values of $\tau$ and for a non-normal distributional assumption for the innovation vectors (see Section 6.5). The additional validation results (not shown for the sake of space) are very similar.

\subsection{Illustration 1: The Impact of the Risk Premium}

In this illustration, we investigate on the impact of the risk premium (reflected in the annualized Sharpe ratio $\lambda$ ) on a longevity hedge. We present two sets of hedging results, one of which is obtained by minimizing the Value-at-Risk of the hedged position in one year, and the other of which is obtained by minimizing the variance of the hedged position in one year. Throughout this sub-section, we assume that the innovation vectors in the model follow a bivariate normal distribution (with the variance-covariance matrix shown in Table 1). This assumption is relaxed in the next sub-section when we study the impact of distributional assumptions.

First, let us examine Figure 8 which shows the relationship between the optimized notional amount $\hat{N}_{1}$ and the annualized Sharpe ratio $\lambda$. When $\lambda=0$, the notional amounts optimized by minimizing Valueat-Risk and minimizing variance are identical. This observation is in accordance to theoretical result in Section 4.4.4: if $\lambda=0$ and the innovation vectors are normally distributed, then minimizing Value-at-Risk and minimizing variance result in the same optimized notional amount.

When $\lambda$ increases, the optimized notional amount obtained by minimizing variance remains unchanged. This outcome is expected, as equation (4.31) indicates that the notional amount optimized by minimizing variance is unrelated to $\lambda$. However, the optimized notional amount obtained by minimizing Value-at-Risk reduces with $\lambda$. This outcome can be explained by considering the following two offsetting effects:

1. When $N_{1}$ increases, the hedge may be more effective in mitigating extreme outcome, thereby reducing the Value-at-Risk.

2. When $N_{1}$ increases, the cost of hedging increases. The increased cost of hedging tends to shift the distribution of the hedged position rightwards and hence raise the Value-at-Risk.

As $\lambda$ increases, the second effect becomes more significant and thus the optimized notional amount reduces. It is interesting to note that at certain values of $\lambda$, the notional amount optimized by minimizing Valueat-Risk becomes negative. This result is because in terms of the quantile of the distribution of the 'hedged position', the 'hedger' may be better off to take more (rather than less) longevity risk if the reward for taking longevity risk (measured by $\lambda$ ) is very attractive. Note also that when $\lambda$ increases further (beyond 4.8), there is no solution to the Value-at-Risk minimization. This outcome confirms our theoretical finding that the solution to Value-at-Risk minimization ceases to exist if $\lambda$ exceeds a certain threshold value. 

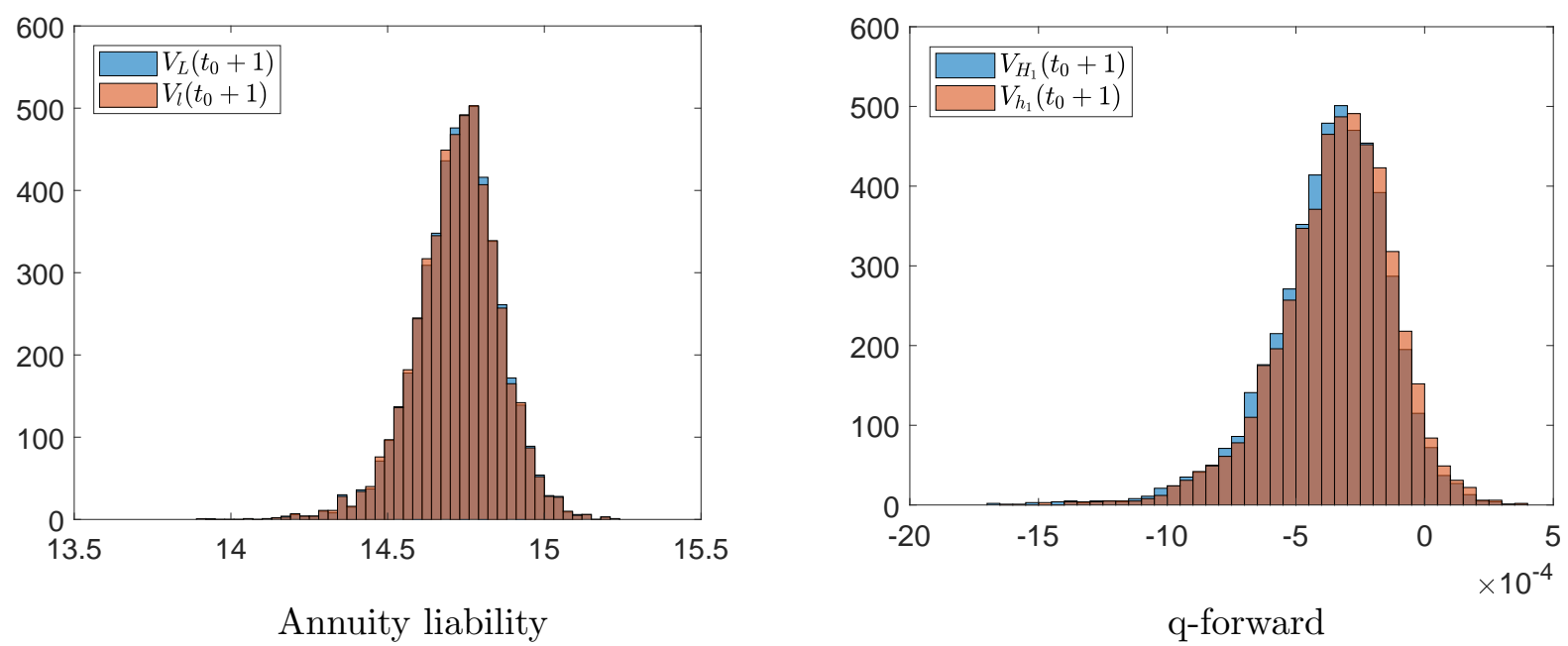

Figure 6: Empirical distributions of $V_{L}\left(t_{0}+1\right), V_{l}\left(t_{0}+1\right), V_{H_{1}}\left(t_{0}+1\right)$ and $V_{h_{1}}\left(t_{0}+1\right)$.

Next, we turn to Figure 9 which displays the relationship between the Value-at-Risk of the hedged position and the annualized Sharpe ratio. As expected, the two optimization objectives lead to the same Value-at-Risk when $\lambda=0$. As $\lambda$ rises, the results from the two minimization objectives diverge, and the benefit (in terms of the Value-at-Risk of the hedged position) of Value-at-Risk minimization over variance minimization becomes more apparent. Consistent with what is observed in Figure 9, there is no solution to the Value-at-Risk minimization when $\lambda>4.8$.

Finally, we study Figure 10 which compares, for different values of $\lambda$, the effectiveness of the longevity hedges using the following metric:

$$
\mathrm{HE}=1-\frac{\operatorname{VaR}_{0.995}\left(V_{L}\left(t_{0}+1\right)-\hat{N}_{1} V_{H_{1}}\left(t_{0}+1\right) \mid \mathcal{F}_{t_{0}}\right)-V_{L}\left(t_{0}\right)}{\operatorname{VaR}_{0.995}\left(V_{L}\left(t_{0}+1\right) \mid \mathcal{F}_{t_{0}}\right)-V_{L}\left(t_{0}\right)}
$$

where $\hat{N}_{1}$ is the optimized notional amount of the q-forward. Loosely speaking, HE represents the fraction of the solvency risk capital that can be reduced by the longevity hedge. A positive value of HE indicates that the hedge benefits the hedger as it reduces the Value-at-Risk of the hedger's portfolio, and vice versa. For the hedge calibrated using Value-at-Risk minimization, the value of HE is always non-negative (provided that $\lambda<4.8$ so that a solution to the optimization problem exists). Nonetheless, for the hedge calibrated using variance minimization, the value of HE becomes negative when $\lambda$ is sufficiently high (greater than 3.5). This undesirable outcome arises because variance minimization focuses only on the dispersion around the location of the distribution of the hedged position but takes no account of the location of the distribution itself. Variance minimization thus fails to take the cost of hedging into account, leads the hedger to acquire an exceedingly large notional amount of q-forward (as cost is ignored), and ultimately results in a Valueat-Risk that may be even higher than that of the unhedged position. This argument is illustrated in Figure 11, which shows the distributions of the hedged positions for $\lambda=0,1,2,3,4$. For all of the five values of $\lambda$, the distributions of the hedged position obtained from variance minimization are equally dispersed. However, as $\lambda$ increases, the distribution shifts to the right, and when $\lambda$ is large enough, the right-tail of the distribution of the hedged position supersedes that of the unhedged position. 


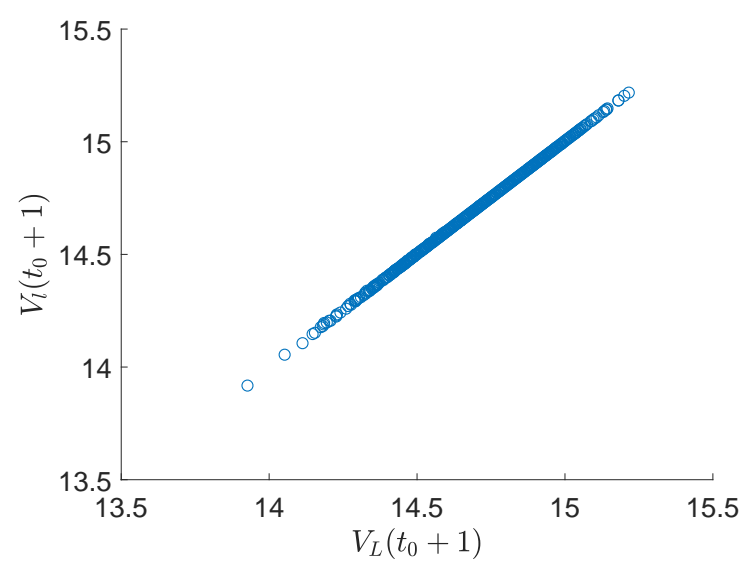

Annuity liability

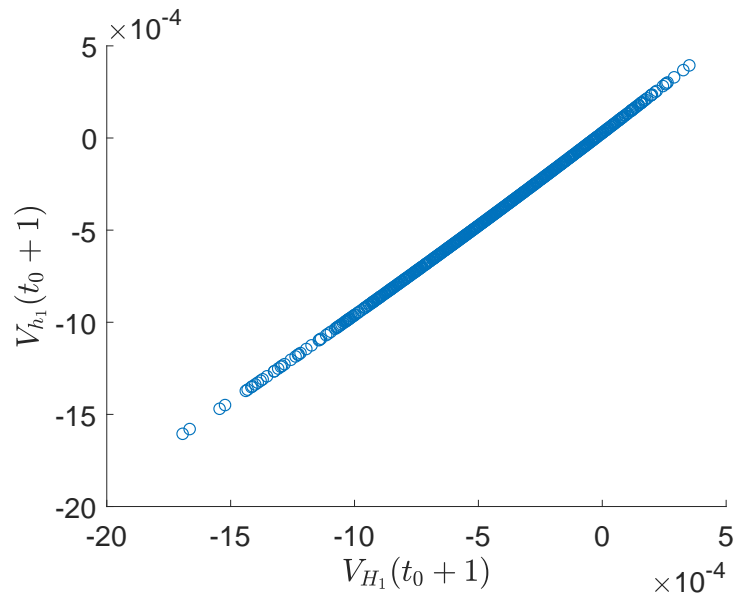

q-forward

Figure 7: Scatter plots of $V_{l}\left(t_{0}+1\right)$ against $V_{L}\left(t_{0}+1\right)$ (left panel) and $V_{h_{1}}\left(t_{0}+1\right)$ against

$$
V_{H_{1}}\left(t_{0}+1\right) \text { (right panel). }
$$

\subsection{Illustration 2: The Impact of Distributional Assumptions}

Figure 12 shows the histograms of the historical values of $\Delta k_{t}^{(1)}$ and $\Delta k_{t}^{(2)}$, which may be regarded as the empirical (marginal) distributions of $\eta_{t}^{(1)}$ and $\eta_{t}^{(2)}$, respectively. It can be observed that the histogram of $\Delta k_{t}^{(1)}$ is right skewed, while that of $\Delta k_{t}^{(2)}$ is left skewed. In the same figure, we also show the estimated normal density functions for $\Delta k_{t}^{(1)}$ and $\Delta k_{t}^{(2)}$, which do not appear to be a close match to the corresponding histograms. The appropriateness of the assumption that the innovations are normally distributed is thus questionable.

We further investigate the appropriateness of the normality assumption by using Henze-Zirkler's multivariate normality test (Henze and Zirkler, 1990), a multivariate normality test that is known for its good power over a wide range of alternatives (Thode, 2002). When applied to the historical values of $\Delta k_{t}^{(1)}$ and $\Delta k_{t}^{(2)}$, the $p$-value of Henze-Zirkler's test is 0.0002444 . This $p$-value suggests that the null hypothesis that $\left(\Delta k_{t}^{(1)}, \Delta k_{t}^{(2)}\right)^{\prime}$ follows a bivariate normal distribution is rejected at a significance level of $0.1 \%$.

We then fit $\left(\Delta k_{t}^{(1)}-\hat{c}^{(1)}, \Delta k_{t}^{(2)}-\hat{c}^{(2)}\right)^{\prime}$ to

$$
\mathrm{St}_{2}(\overrightarrow{0}, \Omega, \vec{\zeta}, \nu)
$$

the bivariate skew- $t$ distribution (with a zero location parameter vector) presented in Section 4.4.3, where $\hat{c}^{(1)}$ and $\hat{c}^{(2)}$ are the sample means of the historical values of $\Delta k_{t}^{(1)}$ and $\Delta k_{t}^{(2)}$, respectively. The parameter estimates are reported in Table 2. The signs of the skewness parameters are in line with the observation that the histogram of $\Delta k_{t}^{(1)}\left(\Delta k_{t}^{(2)}\right)$ is right skewed (left skewed). The fitted (marginal) skew- $t$ density functions for $\Delta k_{t}^{(1)}$ and $\Delta k_{t}^{(1)}$ are also shown in Figure 12. Compared to the normal density functions, the skew- $t$ density functions appear to better resemble their respective empirical distributions.

To study the impact of distributional assumptions, from now on we evaluate longevity hedges using mortality scenarios simulated from the Cairns-Blake-Dowd model of which the innovations follow a bivariate skewed- $t$ distribution with parameters reported in Table 2. Except that concerning the distribution of the innovations, all previously made assumptions are retained. At a fixed annualized Sharpe ratio of $\lambda=3$, 


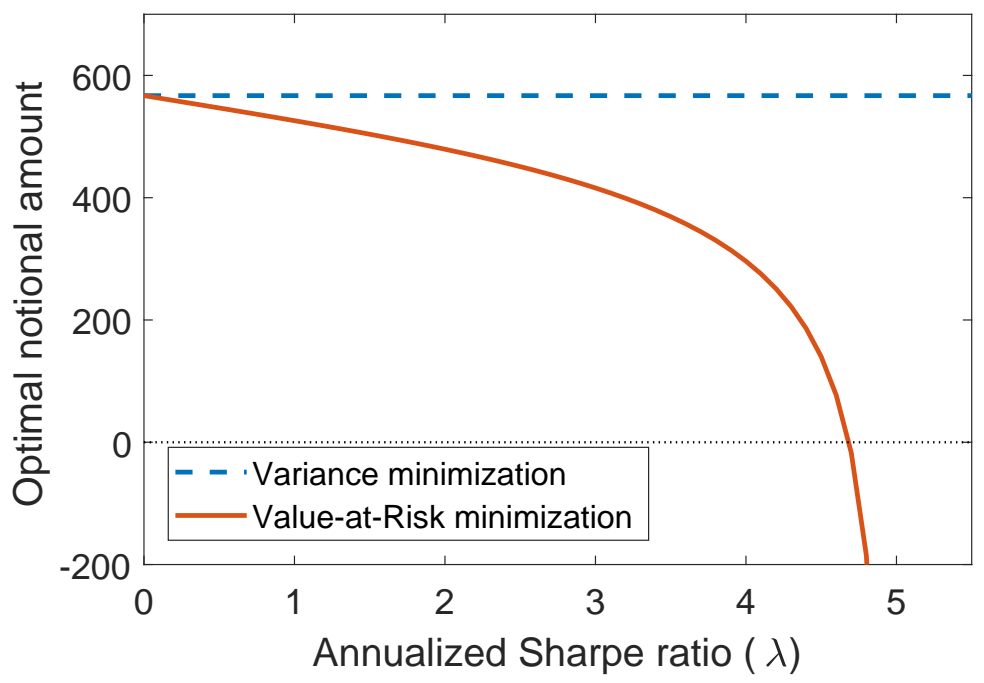

Figure 8: The notional amounts optimized by Value-at-Risk minimization and variance minimization for different values of $\lambda$.

\begin{tabular}{c|c|c}
\hline$\Omega$ & $\nu$ & $\vec{\zeta}$ \\
\hline$\left(\begin{array}{ll}4.3190 \times 10^{-4} & 3.9246 \times 10^{-6} \\
3.9246 \times 10^{-6} & 6.6302 \times 10^{-7}\end{array}\right)$ & 10.0491 & $\left(\begin{array}{c}2.8807 \\
-3.5567\end{array}\right)$ \\
\hline
\end{tabular}

Table 2: Parameter estimates of the bivariate skew- $t$ distribution that is fitted to the historical values of $\left(\Delta k_{t}^{(1)}-\hat{c}^{(1)}, \Delta k_{t}^{(2)}-\hat{c}^{(2)}\right)^{\prime}$.

the Value-at-Risk for each of the following situations is calculated:

1. There is no hedge.

2. A hedge calibrated using variance minimization is used.

3. A hedge calibrated using Value-at-Risk minimization is used. The optimization is performed under the assumption that the innovations follow a bivariate normal distribution, as in Section 6.4. This distributional assumption is overly optimistic, compared to the actual distribution which is skewed and heavier tailed.

4. A hedge calibrated using Value-at-Risk minimization is used. The optimization is performed under the assumption that the innovations follow the bivariate skew- $t$ distribution from which the mortality scenarios for evaluation purposes are generated.

Note that the optimized notional amount for Situation (2) does not depend on the assumed distribution for the innovations.

Figure 13 displays the right tail of the empirical distribution of $V_{L}\left(t_{0}+1\right)$ (the value of the annuity liability at time $t_{0}+1$ ), obtained under the skew- $t$ distributional assumption. In the same figure, we also indicate the Value-at-Risk for each of the four situations described above.

As discussed in Section 4.4.4, if the hedger's objective is to minimize the Value-at-Risk of his/her hedged position, then variance minimization would lead to a sub-optimal hedge if the annualized Sharpe ratio $\lambda$ is greater than zero, and/or the innovations in the assumed mortality are not normally distributed. 


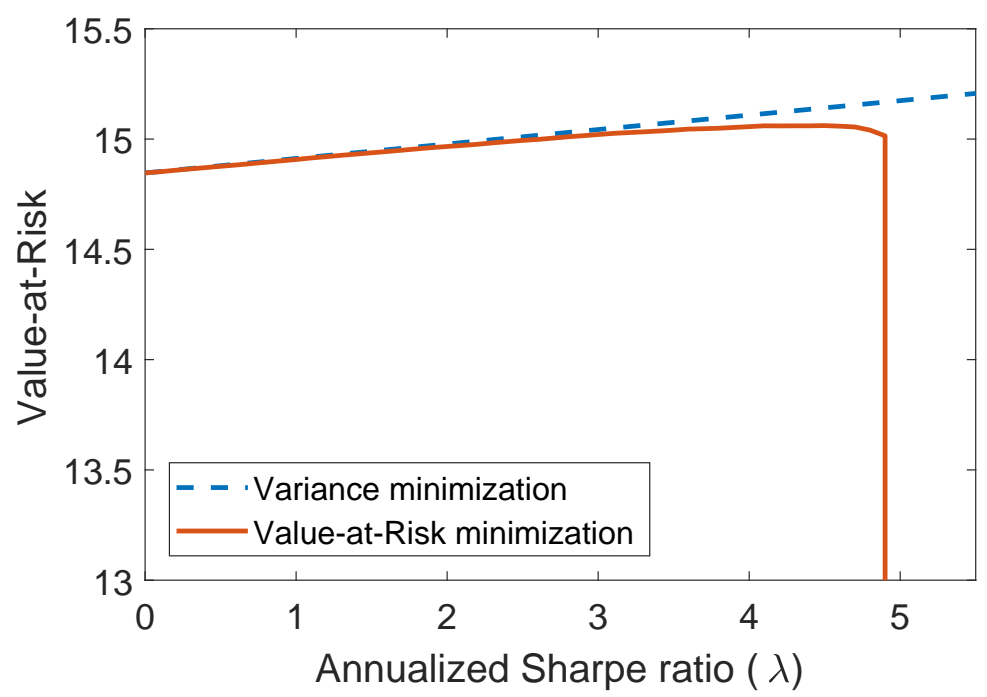

Figure 9: The Value-at-Risk resulting from the hedge optimized by Value-at-Risk minimization and the hedge optimized by variance minimization for different values of $\lambda$.

Here, both conditions apply, and the sub-optimality of variance minimization is clearly manifested in the hedging results: the Value-at-Risk for Situation 2 (a hedge calibrated with variance minimization) is even greater than that for Situation 1 (when there is no hedge).

If the hedge is derived using Value-at-Risk minimization but under an overly optimistic distributional assumption for the innovations, the hedger can still obtain a meaningful reduction in Value-at-Risk. However, the reduction in Value-at-Risk can be made even more significant if the hedger uses a more appropriate distributional assumption for the innovations.

\section{Conclusion}

The $\tau$-year Value-at-Risk is the risk metric that is the most relevant to solvency capital requirements. In this paper, we have proposed a hedging strategy that specifically aims to minimize the $\tau$-year Value-at-Risk of the hedged position. The hedging strategy is built on the general state-space representation of stochastic mortality models, and is therefore applicable to any stochastic mortality model that can be expressed in a state-space form.

When considering Value-at-Risk, it is important not to ignore possible departures from normality. For this reason, we have made our hedging strategy compatible with non-normal distributional assumptions for the innovations in the period and cohort effect processes. For any hedging horizon, the user may assume that the innovations follow a member distribution from the family of stable elliptical distributions. For a hedging horizon of $\tau=1$ year, the user may assume that the innovations follow a member distribution from the family of elliptical distributions or Azzalini and Capitanio's (2003) skew- $t$ distribution. We have also incorporated the costs of the hedging instruments into the derivation of the optimal hedging strategy. The solution of the optimization problem depends on the annualized Sharpe ratio, suggesting that the costs of the hedging instruments play a significant role when the hedger's objective is to minimize the $\tau$-year Value-at-Risk. 


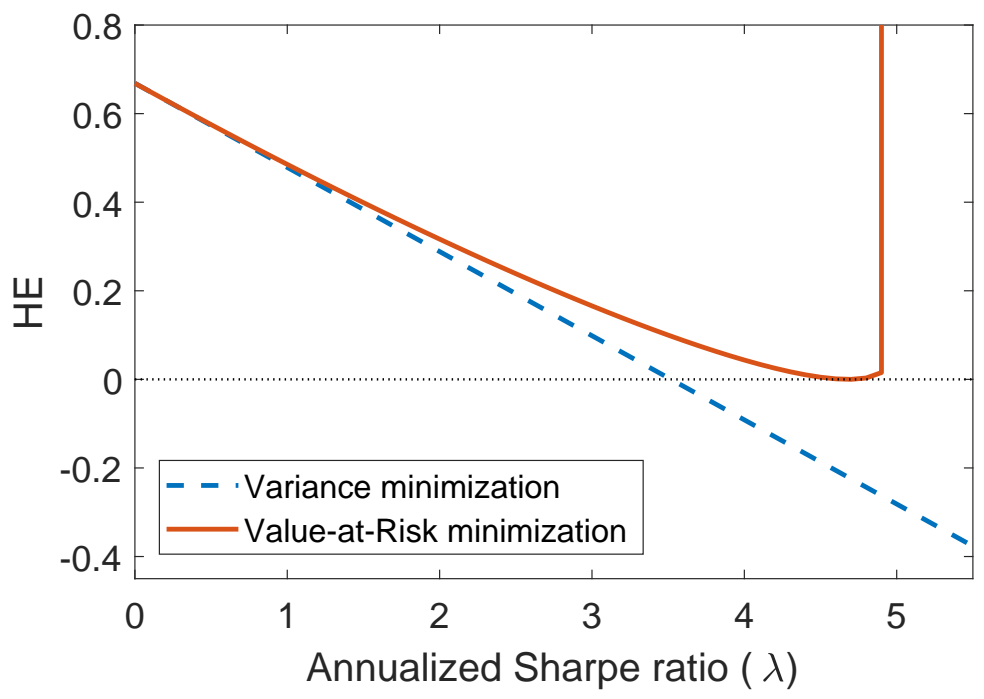

Figure 10: The values of HE resulting from the hedge optimized by Value-at-Risk minimization and the hedge optimized by variance minimization for different values of $\lambda$.

In addition to the hedging strategy, we have contributed a simulation procedure to mitigate the computational burden entailed in calculating the $\tau$-year Value-at-Risk. The procedure eliminates the need for nested simulations by considering (1) the linkage between the hedged position and the innovations in the period and cohort effect processes, and (2) the fact that the innovations are by assumption serially uncorrelated. In contrast to the methods suggested by Cairns (2011) and Plat (2011), our proposed simulation procedure involves no approximation.

We have provided two illustrations using mortality data from the US male population. The illustrations confirm the following facts:

- Value-at-Risk minimization and variance minimization lead to the same optimal hedging strategy when the innovations are normally distributed and the cost of hedging is zero.

- When the annualized Sharpe ratio exceeds a certain threshold value, the solution to the Value-at-Risk minimization problem ceases to exist.

- If the hedger's objective is to minimize Value-at-Risk, a hedge optimized by variance minimization is sub-optimal in general circumstances and may result in a Value-at-Risk that is even higher than the unhedged position.

- A hedge that is derived from a more realistic distributional assumption may be able to offer a better Value-at-Risk reduction.

When incorporating hedge costs into the hedging strategy, we have used the pricing formula proposed by Loeys et al. (2007). Alternatively, for pricing purposes, we may consider a stochastic mortality model that is defined under a risk-neutral probability measure. Future research warrants a study of how a stochastic mortality model in the general state-space form can be 'risk-neutralized'. We remark that the method of Esscher transform, considered previously by Hunt and Blake (2015) for 'risk-neutralizing' certain stochastic mortality models, relies on moment generating functions, and hence does not work when, for example, the 

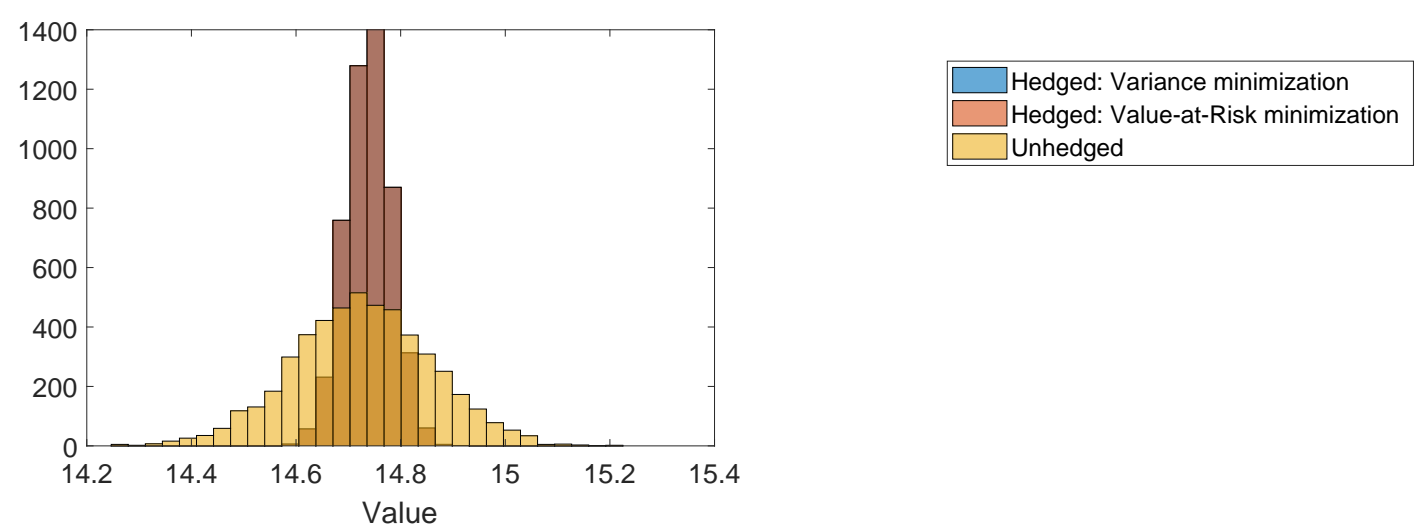

$\lambda=0$
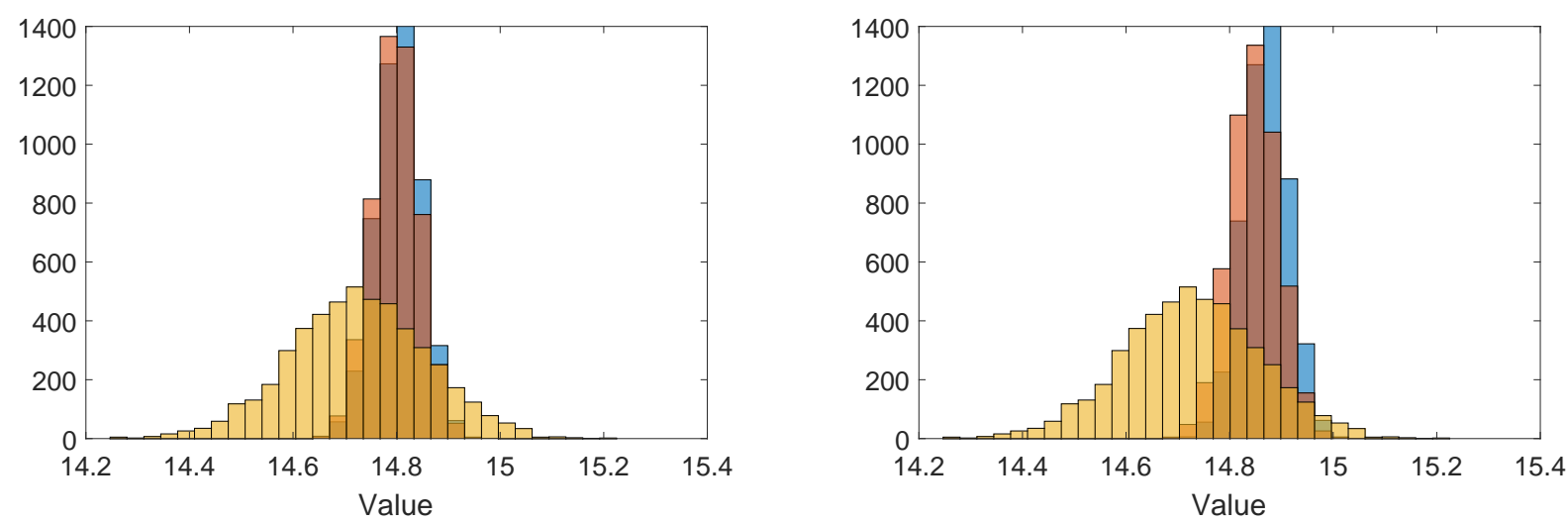

$\lambda=1$
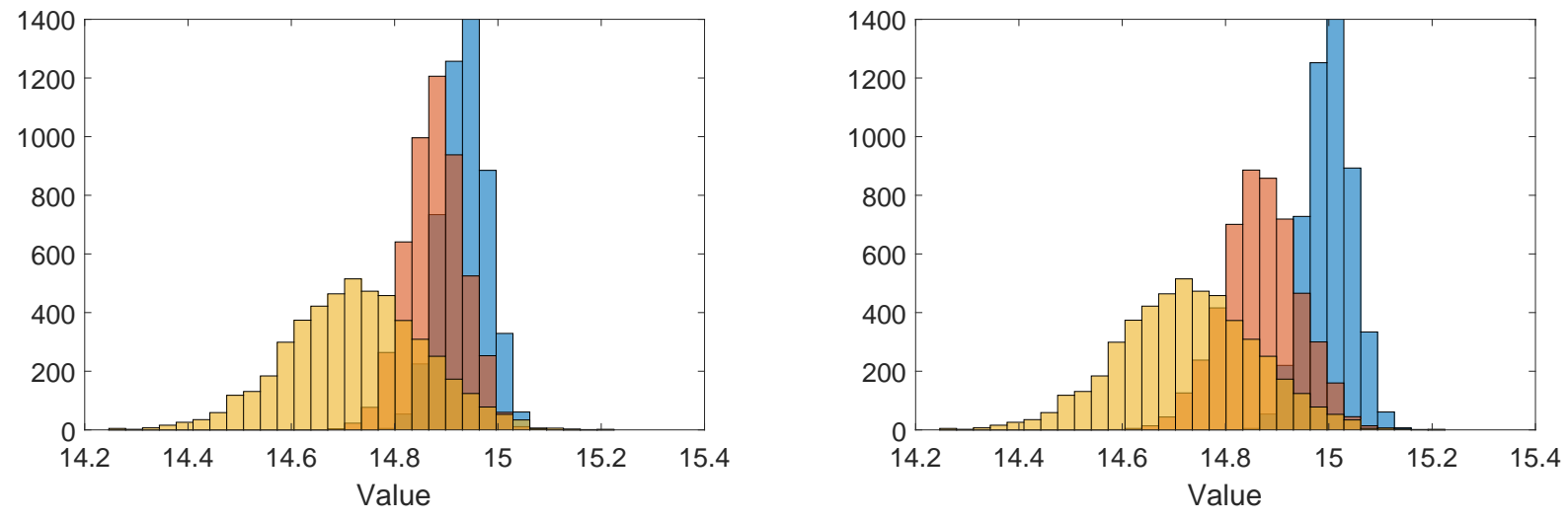

$\lambda=3$

$\lambda=4$

Figure 11: The empirical distributions of (1) the hedged position when the hedge is optimized by Value-at-Risk minimization, (2) the hedged position when the hedge is optimized by variance minimization, and (3) the unhedged position for different values of $\lambda$. 

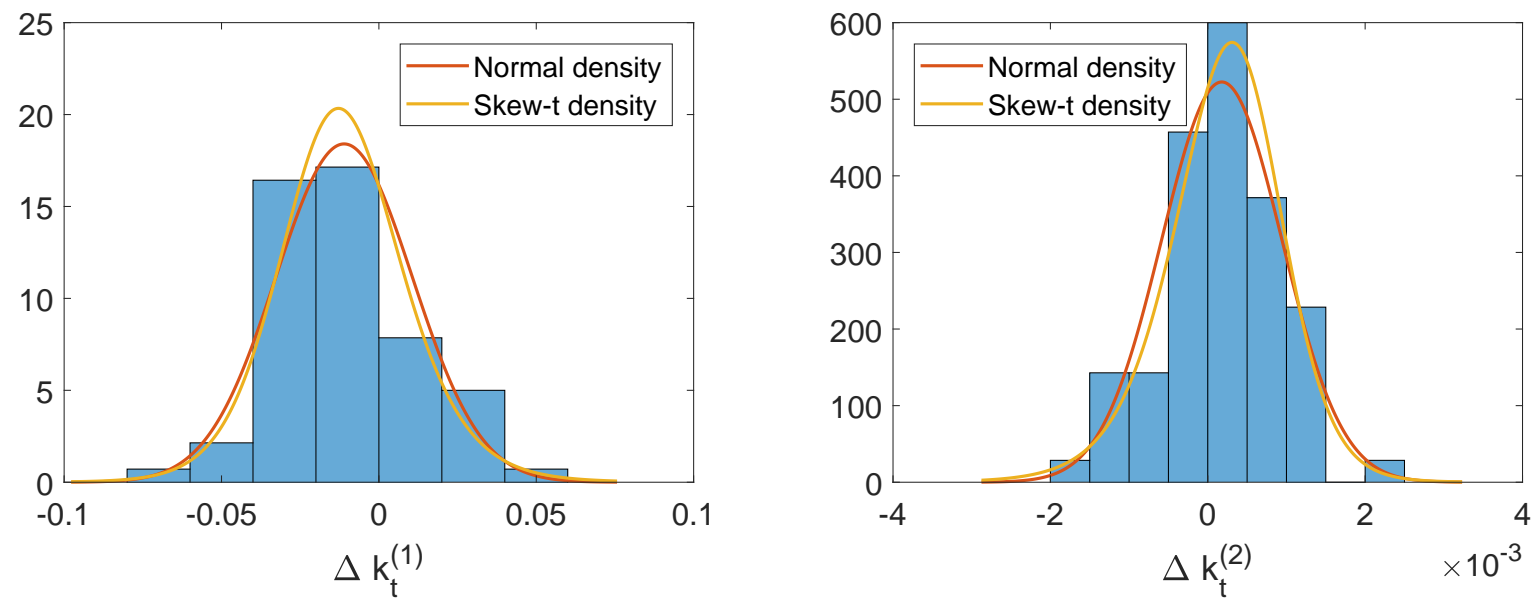

Figure 12: Histograms of the historical values of $\Delta k_{t}^{(1)}$ and $\Delta k_{t}^{(2)}$, and the normal and skew- $t$ density functions that are fitted to the historical values of $\Delta k_{t}^{(1)}$ and $\Delta k_{t}^{(2)}$.

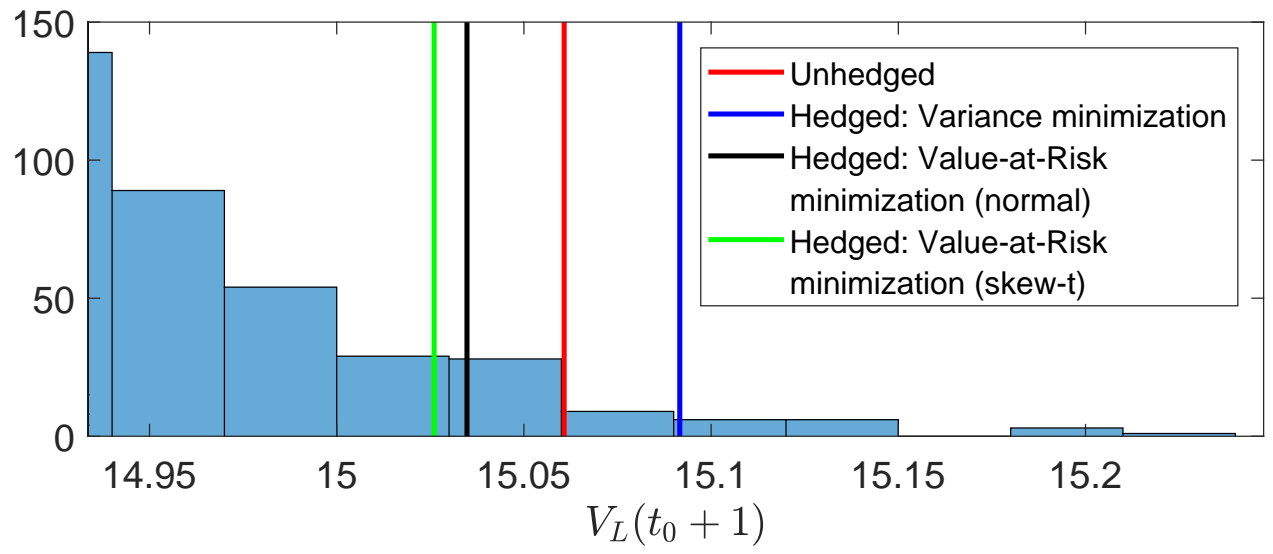

Figure 13: The right-tail of the empirical distribution of $V_{L}\left(t_{0}+1\right)$, and the Value-at-Risk for each of the four situations considered.

innovations are assumed to follow a Student's $t$ distribution of which the moment generating function does not exist.

To focus on our research objectives, we have not incorporated population basis risk (which arises from the difference in mortality experience between the hedger's portfolio of individuals and the population to which the hedging instruments are linked) and trend change risk (which arises from the possibility that the long-term trend in mortality may change over time). Given the flexibility of our set-up, the proposed hedging strategy can be extended rather easily to accommodate these two risks. For the former, we may apply our set-up to a two-population stochastic mortality model that is expressed in a state-space form (see Liu and Li, 2016). For the latter, we may apply our set-up to the LLCBD model (Liu and Li, 2017a), a stochastic mortality model that treats the long-term trend as a random variable and can be expressed in a state-space form straightforwardly.

We have also ignored sampling risk (which arises from the fact that the hedger's portfolio is of a finite size). This risk does not impact the solution to the Value-at-Risk minimization, but affects the calculation 
of the $\tau$-year Value-at-Risk for the hedged and unhedged positions. To incorporate sampling risk, we may simulate death counts using a counting process such as Poisson or 'switch on' the random term (which captures sampling uncertainty) in the observation equation of the assumed model. We remark that it is not always easy to distinguish between sampling risk and trend risk from historical data. Recently, Zhou and $\mathrm{Li}(2017 \mathrm{c})$ has proposed a solution to this issue, and it would be interesting to incorporate their solution into our proposed hedging strategy in future research.

Finally, given the context of this paper, in terms of model selection, we have focused on the distribution of the innovations only. A more comprehensive model selection process should also consider the parametric structure (e.g., the Cairns-Blake-Dowd structure versus the Lee-Carter structure) and the stochastic processes for the period and/or cohort effects in the parametric structure. In future research, we suggest incorporating the choice of distributional assumption into the model selection procedures proposed by Hunt and Blake (2014) and Li et al. (2015).

\section{Acknowledgments}

An earlier version of this paper was presented at the 13th International Longevity Risk and Capital Markets Solutions Conference. The authors are grateful to David Blake, Jeff Mulholland and other participants at the conference for their insightful comments.

This work is supported by research grants from the Global Risk Institute, the Natural Sciences and Engineering Research Council of Canada (Discovery Grant RGPIN-356050-2013) and the Society of Actuaries Center of Actuarial Excellence Program.

\section{References}

Ahmadi, S.S., and Gaillardetz, P. (2014). Two factor stochastic mortality modeling with generalized hyperbolic distribution. Journal of Data Science 12(1): 1-18.

Azzalini, A., and Capitanio, A. (2003). Distributions generated by perturbation of symmetry with emphasis on a multivariate skew $t$-distribution. Journal of the Royal Statistical Society. Series B (Statistical Methodology) 65(2): 367-389.

Azzalini, A., and Capitanio, A. (1999). Statistical applications of the multivariate skew normal distribution. Journal of the Royal Statistical Society. Series B (Statistical Methodology) 61(3): 579-602.

Barrieu, P., Bensusan, H., El Karoui, N., Hillairet, C., Loisel, S., Ravanelli, C., and Salhi, Y. (2012). Understanding, modelling and managing longevity risk: Key issues and main challenges. Scandinavian Actuarial Journal 2012(3): 203-231.

Bauer, D., Börger, M., and Ruß, J. (2010). On the pricing of longevity-linked securities. Insurance: Mathematics and Economics, 46(1), 139-149.

Blake, D., Boardman, T., and Cairns, A. (2014). Sharing longevity risk: Why governments should issue longevity bonds. North American Actuarial Journal 18(1): 258-277.

Börger, M. (2010). Deterministic shock vs. stochastic value-at-riskan analysis of the Solvency II standard model approach to longevity risk. Blätter der DGVFM 31(2): 225-259. 
Börger, M., Fleischer, D., and Kuksin, N. (2014). Modeling the mortality trend under modern solvency regimes. ASTIN Bulletin 44(1): 1-38.

Boyer, M.M., Mejza, J., and Stentoft, L. (2014). Measuring longevity risk: An application to the Royal Canadian Mounted Police pension plan. Risk Management and Insurance Review 17(1): 37-59.

Cairns, A.J.G. (2013). Robust hedging of longevity risk. Journal of Risk and Insurance 80(3): 621-648.

Cairns, A.J.G. (2011). Modelling and management of longevity risk: Approximations to survivor functions and dynamic hedging. Insurance: Mathematics and Economics 49(3): 438-453.

Cairns, A.J.G., Blake, D., and Dowd, K. (2006). A twofactor model for stochastic mortality with parameter uncertainty: Theory and calibration. Journal of Risk and Insurance 73(4): 687-718.

Cairns, A.J.G., Blake, D., Dowd, K., Coughlan, G.D., Epstein, D., Ong, A., and Balevich, I. (2009). A Quantitative Comparison of Stochastic Mortality Models Using Data from England and Wales and The United States. North American Actuarial Journal 13: 1-35.

Coughlan, G.D., Khalaf-Allah, M., Ye, Y., Kumar, S., Cairns, A.J., Blake, D., and Dowd, K. (2011). Longevity hedging 101: A framework for longevity basis risk analysis and hedge effectiveness. North American Actuarial Journal 15(2): 150-176.

Dowd, K., Cairns, A.J.G., and Blake, D. (2006). Mortality-dependent financial risk measures. Insurance: Mathematics and Economics 38(3): 427-440.

Fung, M.C., Peters, G.W., and Shevchenko, P. V. (2017). A unified approach to mortality modelling using state-space framework: Characterisation, identification, estimation and forecasting. Annals of Actuarial Science 11: 343-389.

Fujisawa, Y., and Li, J.S.-H. (2012). The impact of the automatic balancing mechanism for the public pension in Japan on the extreme elderly. North American Actuarial Journal 16(2), 207-239.

Fujisawa, Y., and Li, J.S.-H. (2010). IFRS convergence: The role of stochastic mortality models in the disclosure of longevity risk for defined benefit plans. Asia-Pacific Journal of Risk and Insurance $5(1)$.

Graziani, G. (2014). Longevity risk - A fine balance. Institutional Investor Journals: Special Issue on Pension and Longevity Risk Transfer for Institutional Investors 2014: 35-27.

Hunt, A., and Blake, D. (2015). Forward mortality rates in discrete time I: Calibration and securities pricing. Pensions Institute Discussion Paper PI-1511.

Hunt, A., and Blake, D. (2014). A general procedure for constructing mortality models. North American Actuarial Journal 18(1): 116-138.

Lee, R. D., and Carter, L. R. (1992). Modeling and forecasting US mortality. Journal of the American statistical association 87(419): 659-671.

Levantesi, S., and Menzietti, M. (2017). Maximum market price of longevity risk under solvency regimes: The case of solvency II. Risks 5(2): 29.

Li, J.S.-H., and Hardy, M.R. (2011). Measuring basis risk in longevity hedges. North American Actuarial Journal 15(2): 177-200. 
Li, J.S.-H., and Luo, A. (2012). Key q-duration: A framework for hedging longevity risk. ASTIN Bulletin 42(2): 413-452.

Li, J.S.H., Zhou, R. and Hardy, M. (2015) A step-by-step guide to building two-population stochastic mortality models. Insurance: Mathematics and Economics 63: 121-134.

Lin, T. and Tsai, C.C.L. (2013). On the mortality/longevity risk hedging with mortality immunization. Insurance: Mathematics and Economics 53(3): 580-596.

Liu, Y., and Li, J.S.-H. (2017a). The locally linear Cairns-Blake-Dowd model: A note on delta-nuga hedging Of longevity risk. ASTIN Bulletin 47(1): 79-151.

Liu, Y., and Li, J.S.-H. (2017b). A strategy for hedging risks associated with period and cohort effects using q-forwards. Insurance: Mathematics and Economics, in press.

Liu, Y., and Li, J.S.-H. (2016). It's all in the hidden states: A longevity hedging strategy with an explicit measure of population basis risk. Insurance: Mathematics and Economics 70: 301-319.

Loeys, J., Panigirtzoglou, N., and Ribeiro, R. (2007). Longevity: A market in the making. J.P. Morgan Research Paper.

Michaelson, A., and Mulholland, J. (2014). Strategy for increasing the global capacity for longevity risk transfer: Developing transactions that attract capital markets investors. Journal of Alternative Investments 17: 18-27.

Olivieri, A., and Pitacco, E. (2009). Stochastic mortality: The impact on target capital. ASTIN Bulletin 39(2): 541-563.

Plat, R. (2011). One-year value-at-risk for longevity and mortality. Insurance: Mathematics and Economics 49(3): 462-470.

Plat, R. (2009a). Stochastic portfolio specific mortality and the quantification of mortality basis risk. Insurance: Mathematics and Economics 45(1): 123-132.

Plat, R., (2009b). On stochastic mortality modeling. Insurance: Mathematics and Economics 45: 393404.

Renshaw, A. E., and Haberman, S. (2003). LeeCarter mortality forecasting with age-specific enhancement. Insurance: Mathematics and Economics 33(2): 255-272.

Richards, S.J., Currie, I.D., and Ritchie, G. P. (2014). A Value-at-Risk framework for longevity trend risk. British Actuarial Journal 19(1): 116-139.

Ngai, A., and Sherris, M. (2011). Longevity risk management for life and variable annuities: The effectiveness of static hedging using longevity bonds and derivatives. Insurance: Mathematics and Economics 49(1): 100-114.

Theodossiou, P. (1998). Financial data and the skewed generalized t distribution. Management Science 44(12-part-1): 1650-1661.

Thode, H.C. (2002). Testing for normality (Vol. 164). CRC press.

Wang, C.W., Huang, H.C., and Liu, I.C. (2011). A quantitative comparison of the Lee-Carter model under different types of non-Gaussian innovations. The Geneva Papers on Risk and Insurance Issues and Practice 36(4): 675-696. 
Zhang, J., Tan, K.S., and Weng, C. (2017). Optimal dynamic longevity hedge with basis risk. Working paper.

Zhou, K.Q. and Li, J.S.-H. (2017a). Longevity Greeks: What do insurers and capital market investors need to know? North American Actuarial Journal, in press.

Zhou, K.Q. and Li, J.S.-H. (2017b). Dynamic longevity hedging in the presence of population basis risk: A feasibility analysis from technical and economic perspectives. Journal of Risk and Insurance 84(S1): 417-437.

Zhou, K.Q. and Li, J.S.-H. (2017c). Improving the distinction between sampling risk and trend risk: A Fay-Herriot extension of stochastic mortality models. Paper presented at the 13th International Longevity Risk and Capital Markets Solutions Conference, Taipei, Taiwan ROC.

Zhou, R., Wang, Y., Kaufhold, K., Li, J.S.-H., and Tan, K.S. (2014). Modeling period effects in multipopulation mortality models: Applications to Solvency II. North American Actuarial Journal 18(1), 150-167. 